\title{
Sharp linear and bilinear restriction estimates for paraboloids in the cylindrically symmetric case
}

Shuanglin Shao

\begin{abstract}
For cylindrically symmetric functions dyadically supported on the paraboloid, we obtain a family of sharp linear and bilinear adjoint restriction estimates. As corollaries, we first extend the ranges of exponents for the classical linear or bilinear adjoint restriction conjectures for such functions and verify the linear adjoint restriction conjecture for the paraboloid. We also interpret the restriction estimates in terms of solutions to the Schrödinger equation and establish the analogous results when the paraboloid is replaced by the lower third of the sphere.
\end{abstract}

\section{Introduction}

Let $n \geq 3$ be a fixed integer and $S$ be a smooth compact non-empty subset of the paraboloid $\left\{(\tau, \xi) \in \mathbb{R} \times \mathbb{R}^{n-1}: \tau=|\xi|^{2}\right\}$. If $0<p, q \leq \infty$, the classical linear adjoint restriction estimate ${ }^{1}$ for the paraboloid is the a priori estimate

$$
\left\|(g d \sigma)^{\vee}\right\|_{L_{t, x}^{q}\left(\mathbb{R} \times \mathbb{R}^{n-1}\right)} \leq C_{p, q, n, S}\|g\|_{L^{p}(S, d \sigma)}
$$

for all Schwartz functions $g$ on $S$, where

$$
(g d \sigma)^{\vee}(t, x)=\int_{S} g(\tau, \xi) e^{i(x \cdot \xi+t \tau)} d \sigma(\xi)=\int_{\mathbb{R}^{n-1}} g\left(|\xi|^{2}, \xi\right) e^{i\left(x \cdot \xi+t|\xi|^{2}\right)} d \xi
$$

denotes the inverse space-time Fourier transform of the measure $g d \sigma$, and $d \sigma$ is the canonical measure of the paraboloid defined in Section 2.

2000 Mathematics Subject Classification: Primary 42B10, 42B25; Secondary 35Q55. Keywords: Restriction conjecture, Schrödinger equation, cylindrical symmetry.

${ }^{1}$ In the notation of [17], the estimate $(1.1)$ is denoted by $R_{S}^{*}(p \rightarrow q)$ and the estimate $(1.2)$ is denoted by $R_{S_{1}, S_{2}}^{*}(p \times p \rightarrow q)$. 
By duality, the estimate (1.1) is equivalent to the following estimate

$$
\|\hat{f}\|_{L^{p^{\prime}(S, d \sigma)}} \leq C_{p, q, n, S}\|f\|_{L^{q^{\prime}\left(\mathbb{R} \times \mathbb{R}^{n-1}\right)}}
$$

for all Schwartz functions $f$, which roughly says that the Fourier transform of an $L^{q^{\prime}}\left(\mathbb{R} \times \mathbb{R}^{n-1}\right)$ function can be "meaningfully" restricted to the paraboloid $S$. This leads to the restriction problem, one of the central problems in harmonic analysis, which concerns the optimal range of exponents $p$ and $q$ for which the estimate (1.1) should hold. It was originally proposed by Stein for the sphere [13] and then extended to smooth sub-manifolds of $\mathbb{R} \times \mathbb{R}^{n-1}$ with appropriate curvature [14] such as the paraboloid and the cone. The restriction problem is intricately related to other outstanding problems in analysis such as the Bochner-Riesz conjecture, the local smoothing conjecture, the Kakeya set conjecture and the Kakeya maximal function conjecture, see e.g., [17], [18].

In this paper, we will mainly focus on the restriction estimates for the paraboloid. The corresponding linear adjoint restriction conjecture for the paraboloid asserts that

Conjecture 1.1. The inequality (1.1) holds with constants depending on $S$, $n$ and $p, q$ if and only if $q>\frac{2 n}{n-1}$ and $\frac{n+1}{q} \leq \frac{n-1}{p^{\prime}}$.

The conditions on $p$ and $q$ are known to be best possible by the decay estimates of $(d \sigma)^{\vee}$ and the standard Knapp example, see e.g., [14], [17]. When $n=2$, the non-endpoint case was first proven to be true by Fefferman and Stein [7] (and generalized to other oscillatory integrals by Carleson and Sjölin [5]), and the endpoint case was proven to be true by Zygmund [29]. When $n>2$, it was proven with the additional condition $q>\frac{2(n+1)}{n-1}$ by Tomas [25] using real interpolation, and $q=\frac{2(n+1)}{n-1}$ by Stein [14] using complex interpolation. In 1977, Córdoba [6] gave an alternate proof for $n=2$ by largely relying on the successful resolution of the Kakeya conjecture in two dimensions. In 1991, Bourgain [1] generalized Córdoba's arguments to higher dimensions, so that nontrivial progress on the Kakeya problem might imply some nontrivial progress on the restriction result; using this technique, he proved estimates for some $q<\frac{2(n+1)}{n-1}$; in particular, $q>4-\frac{2}{15}$ when $n=3$. Further improvements along this line were made by Moyua, Vargas, Vega and Wolff, see e.g., [11], [27]. The current best result $q>\frac{2(n+2)}{n}$ in higher dimensions $n \geq 3$ is due to Tao [20], based on the techniques in Wolff's breakthrough paper on the cone restriction estimates [28].

Among various techniques developed to attack this problem, the bilinear method proves to be very powerful. Variants of this idea also have applications to the nonlinear dispersive equations, see e.g., [2], [3], [9], etc. More 
precisely, we assume $S_{1}$ and $S_{2}$ to be two smooth compact non-empty subsets of the paraboloid in $\mathbb{R} \times \mathbb{R}^{n-1}$, which are transverse in the sense that the unit normals of $S_{1}$ and of $S_{2}$ are separated by at least some fixed angle $c>0$. Then the classical bilinear adjoint restriction conjecture concerns the optimal range of exponents $p$ and $q$ for which the bilinear operator, $(f, g) \rightarrow\left(f d \sigma_{1}\right)^{\vee}\left(g d \sigma_{2}\right)^{\vee}$, should bound from $L^{p} \times L^{p}$ to $L^{q}$, where $d \sigma_{1}, d \sigma_{2}$ are the canonical Lebesgue measures of $S_{1}, S_{2}$, respectively. The following formulation of this conjecture is taken from [23].

Conjecture 1.2. Let $S_{1}, S_{2}$ be defined as above and $q \geq \frac{n}{n-1}, \frac{n+2}{2 q}+\frac{n}{p} \leq n$ and $\frac{n+2}{2 q}+\frac{n-2}{p} \leq n-1$. Then there exists a constant $0<C<\infty$ depending on $S_{1}, S_{2}, n$ and $p, q$ such that

$$
\left\|\left(f d \sigma_{1}\right)^{\vee}\left(g d \sigma_{2}\right)^{\vee}\right\|_{L_{t, x}^{q}\left(\mathbb{R} \times \mathbb{R}^{n-1}\right)} \leq C\|f\|_{L^{p}\left(S_{1}\right)}\|g\|_{L^{p}\left(S_{2}\right)}
$$

for all $f \in L^{p}\left(S_{1}\right)$ and $g \in L^{p}\left(S_{2}\right)$.

It is known that the bilinear restriction conjecture 1.2 is stronger than the linear restriction conjecture 1.1, see [23]. For a discussion of recent progress made on this problem, see [17]. We remark that the conditions on $p$ and $q$ in this conjecture are also known to be best possible by the decay estimates of $(d \sigma)^{\vee}$ and the variants of the standard Knapp examples such as the squashed caps and the stretched caps, see e.g., [23], [17].

However, none of these Knapp-type examples are cylindrically symmetric functions, i.e., functions on $\mathbb{R} \times \mathbb{R}^{n-1}$ invariant under spatial rotations. Hence we expect that further estimates are available if we assume that functions are cylindrically symmetric and supported on a dyadic subset of the paraboloid in the form of $\left\{(\tau, \xi): M \leq|\xi| \leq 2 M, \tau=|\xi|^{2}\right\}$ with $M \in 2^{\mathbb{Z}}$. We denote by $\mathcal{L}_{M}$ this class of functions. Indeed, it is the case: when $n=3$, the Tomas-Stein restriction estimate $L^{2} \rightarrow L^{4}$ is known to be best possible; but for functions in $\mathcal{L}_{M}$, the estimate $L^{2} \rightarrow L^{q}$ is true for any $q>10 / 3$ by Corollary 2.3 in Section 3.

Our main theorems, Theorem 2.1 and 2.5, of this paper are to present a family of sharp linear adjoint restriction estimates for $f \in \mathcal{L}_{1}$, and bilinear ones for $f \in \mathcal{L}_{1}$ and $g \in \mathcal{L}_{M}$ with $0<M \leq 1 / 4$ on the dyadic space-time slab $\mathbb{R} \times\{R / 2 \leq|x| \leq R\}$ with $R \in 2^{\mathbb{Z}}$. The proofs essentially combine the two classical and elementary methods, the Carleson-Sjölin argument [14] and the bilinear method via the Whitney decomposition, which effectively solved the two dimensional restriction conjecture. In the arguments, we heavily exploit the rotational symmetry via the "Fourier-Bessel" formula, Lemma 3.2, for cylindrically symmetric functions to reduce matters to main term estimates by encoding the error term into certain integrals. A lot of effort is devoted to inventing counterexamples to show that the restriction 
estimates are best possible by relying on the idea coming from the standard Knapp examples, the principles of both stationary phase and non-stationary phase [14] and the Khintchine inequality [17]. We remark that some of of them are quite challenging, see e.g., Example 4.5.

As corollaries of the main theorems, we can verify the inequality (1.1) for $\mathcal{L}_{M}$ when the exponents $p$ and $q$ are in a larger region (see Figure 2) and show that it is nearly sharp except for certain endpoints. Furthermore, we show that the linear adjoint restriction conjecture 1.1 holds for all cylindrically symmetric functions when $p$ and $q$ are restricted to the classical region. By similar arguments, one can also establish the analogous sharp restriction estimates when the paraboloid is replaced by the lower third of the sphere $\mathcal{S}^{n-1}$ or more general cylindrically symmetric and compact hypersurfaces of elliptic type as defined in [12], [23]. As applications of the restriction estimates, we will interpret them in terms of the solutions to the Schödinger equations and present another proof of the weighted Strichartz estimates in [26] for Schrödinger equations.

Acknowledgements. The author is very grateful to his advisor Terence Tao for introducing this fascinating subject, and is indebted to him for many helpful conversations and encouragement during the preparation of this paper. The author thanks Monica Visan for helpful discussions. The author would like to thank the referee for his valuable comments and suggestions.

\section{Notations and Main Theorems}

Let $n \geq 3$ be the fixed space-time dimension. In this paper, we interpret $\mathbb{R} \times \mathbb{R}^{n-1}$ as the space-time frequency space.

We will use the notations $X \lesssim Y, Y \gtrsim X$, or $X=O(Y)$ to denote the estimate $|X| \leq C Y$ for some constant $0<C<\infty$, which may depend on $p, q, n$ and $S_{1}$ or $S_{2}$, but not on the functions. If $X \lesssim Y$ and $Y \lesssim X$ we will write $X \sim Y$. If the constant $C$ depends on a special parameter other than the above, we shall denote it explicitly by subscripts. For example, $C_{\varepsilon}$ should be understood as a positive constant not only depending on $p, q, n$ and $S_{1}$ or $S_{2}$, but also on $\varepsilon$.

We denote by $d \sigma$ the canonical Lebesgue measure of the standard paraboloid $S=\left\{(\tau, \xi): \tau=|\xi|^{2}\right\}$ in $\mathbb{R} \times \mathbb{R}^{n-1}$, which is the pullback of $n-1$ dimensional Lebesgue measure $d \xi$ under the projection map $(\tau, \xi) \mapsto \xi$; thus,

$$
\int_{S} f(\tau, \xi) d \sigma=\int_{\mathbb{R}^{n-1}} f\left(|\xi|^{2}, \xi\right) d \xi
$$

By $\mathcal{S}^{n-2}$ we denote the $n-1$ dimensional unit sphere canonically embedded in $\mathbb{R}^{n-1}$, and by $d \mu$ its surface measure. 
We define a dyadic number to be any number $R \in 2^{\mathbb{Z}}$ of the form $R=2^{j}$ where $j$ is an integer. For each dyadic number $R>0$, we define the dyadic annulus in $\mathbb{R}^{n-1}$,

$$
A_{R}:=\left\{x \in \mathbb{R}^{n-1}: R / 2 \leq|x| \leq R\right\} .
$$

We define the space-time norm $L_{t}^{q} L_{x}^{r}$ of $f$ on $\mathbb{R} \times \mathbb{R}^{n-1}$ by

$$
\|f\|_{L_{t}^{q} L_{x}^{r}\left(\mathbb{R} \times \mathbb{R}^{n-1}\right)}=\left(\int_{\mathbb{R}}\left(\int_{\mathbb{R}^{n-1}}|f(t, x)|^{r} d x\right)^{q / r} d t\right)^{1 / q},
$$

with the usual modifications when $q$ or $r$ are equal to infinity, or when the domain $\mathbb{R} \times \mathbb{R}^{n-1}$ is replaced by a small region of space-time such as $\mathbb{R} \times A_{R}$. When $q=r$, we abbreviate it by $L_{t, x}^{q}$. Unless specified in this paper, all the space-time integrals are taken over $\mathbb{R} \times A_{R}$ with dyadic $R>0$, which will be clear from the context.

We define the spatial Fourier transform of $f$ on $\mathbb{R}^{n-1}$ by

$$
\hat{f}(\xi)=\int_{\mathbb{R}^{n-1}} f(x) e^{-i x \cdot \xi} d x .
$$

We use $1_{U}$ to denote the indicator function of the set $U$, i.e.,

$$
1_{U}(x)= \begin{cases}1, & \text { for } x \in U \\ 0, & \text { for } x \notin U\end{cases}
$$

For $1 \leq p \leq \infty$, we denote the conjugate exponent of $p$ by $p^{\prime}$, i.e., $1 / p+$ $1 / p^{\prime}=1$.

We start with stating the main theorem concerning the linear restriction estimates, which is proven in Section 3.

Theorem 2.1. Suppose $f \in \mathcal{L}_{1}$ and $R>0$ is a dyadic number. Then the following sharp restriction estimates hold:

- $\operatorname{for} q=2$ and $2 \leq p \leq \infty$,

$$
\left\|(f d \sigma)^{\vee}\right\|_{L_{t, x}^{2}} \lesssim \min \left\{R^{\frac{1}{2}}, R^{\frac{n-1}{2}}\right\}\|f\|_{L^{p}(S)} .
$$

- for $q=4$ and $4 \leq p \leq \infty, \forall \varepsilon>0$,

$$
\left\|(f d \sigma)^{\vee}\right\|_{L_{t, x}^{4}} \lesssim_{\varepsilon} \min \left\{R^{-\frac{n-2}{4}+\varepsilon}, R^{\frac{n-1}{4}}\right\}\|f\|_{L^{p}(S)} .
$$

- for $q=3 p^{\prime}$ and $1 \leq p<4$,

$$
\left\|(f d \sigma)^{\vee}\right\|_{L_{t, x}^{q}} \lesssim \min \left\{R^{(n-2)\left(\frac{1}{q}-\frac{1}{2}\right)}, R^{\frac{n-1}{q}}\right\}\|f\|_{L^{p}(S)} .
$$


- for $q=\infty$ and $1 \leq p \leq \infty$,

$$
\left\|(f d \sigma)^{\vee}\right\|_{L_{t, x}^{\infty}} \lesssim \min \left\{R^{-\frac{n-2}{2}}, 1\right\}\|f\|_{L^{p}(S)}
$$

where $A$ in $\{A, B\}$ is given by the case where $R \geq 2$ and $B$ by $R \leq 1$. Furthermore, by interpolation we obtain the sharp restriction estimates $L^{p} \rightarrow L^{q}$ when $p, q$ are in the region determined by these lines (Figure 1).

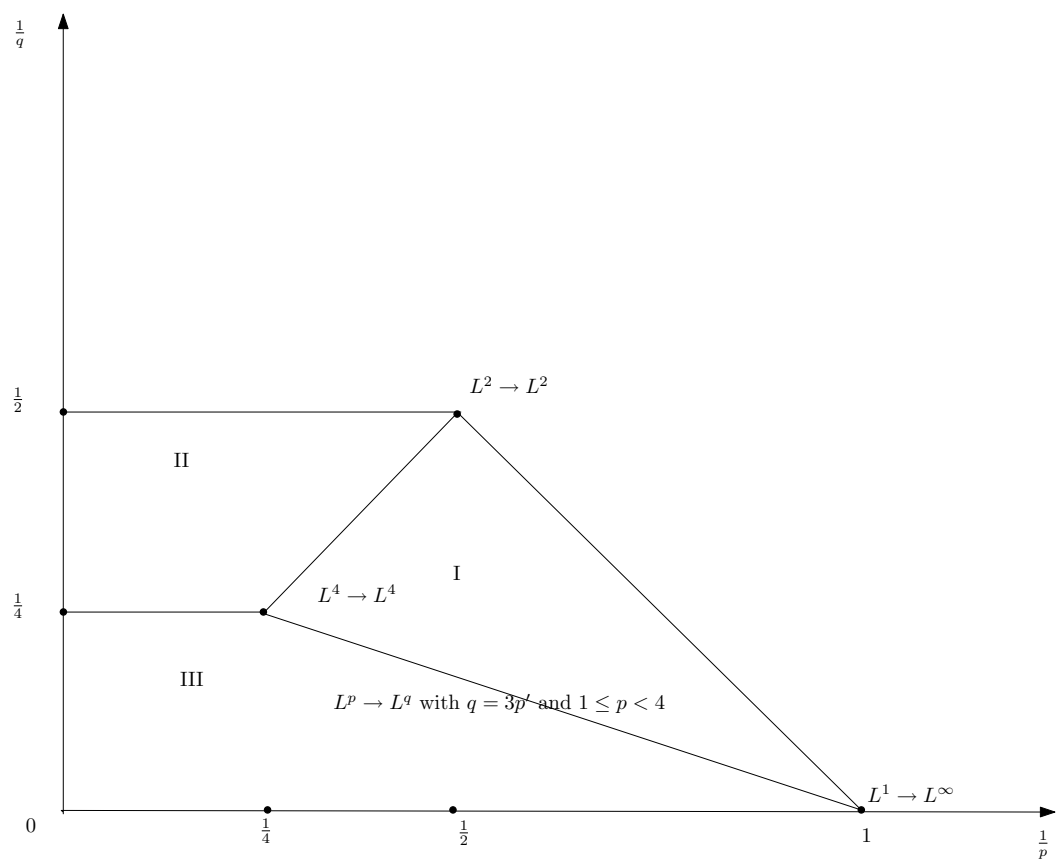

Figure 1: Linear restriction estimates on $\mathbb{R} \times A_{R}$.

Remark 2.2. We observe that the estimates above in each case are "continuous" in the sense that they match when $R \sim 1$.

One can easily obtain the following corollary regarding the linear adjoint restriction conjecture.

Corollary 2.3. Suppose $f$ are cylindrically functions supported on the paraboloid.

- If $f \in \mathcal{L}_{M}$, the linear adjoint restriction conjecture 1.1 holds with constants depending on $p, q, n$ and $M$ whenever $q>\frac{2 n}{n-1}, \frac{1}{p}+\frac{1}{q} \leq 1$ and $\frac{1}{p}+\frac{2 n-1}{q}<n-1$ (Figure 2 ).

- The linear adjoint restriction conjecture 1.1 is true for all $f$ (Figure 2). 


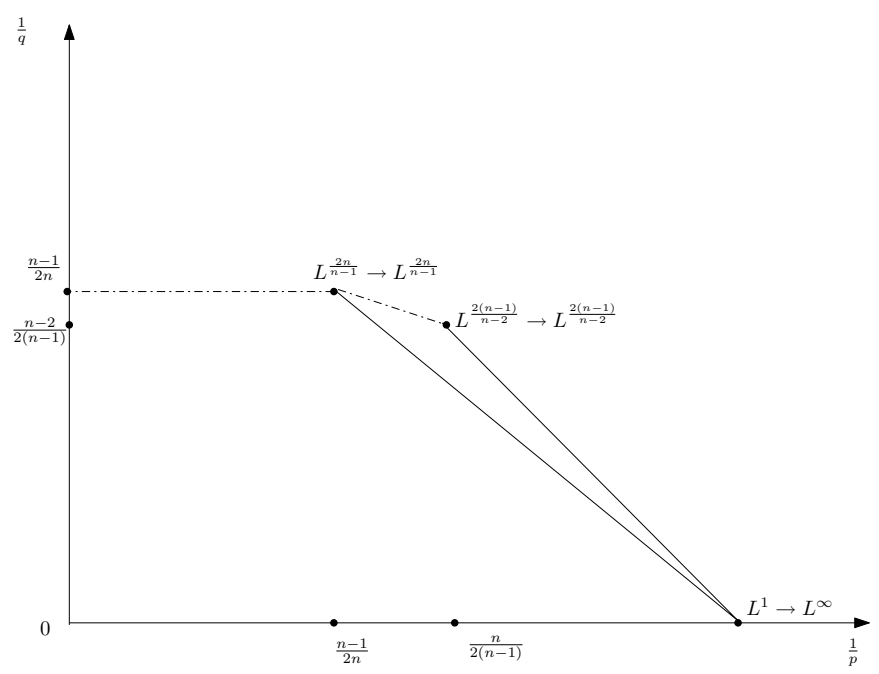

Figure 2: Linear restriction estimates for $\mathcal{L}_{M}$ (cf. the inside trapezoid, the classical range of $p$ and $q$ for Conjecture 1.1).

Proof. By Theorem 2.1, the first assertion follows from scaling to $f \in \mathcal{L}_{1}$ and then summing all dyadic $R$ and using interpolation.

To prove the second assertion, it is sufficient to obtain it under the boundary conditions $q>\frac{2 n}{n-1}$ and $\frac{n+1}{q}=\frac{n-1}{p^{\prime}}$ since other estimates are easily obtained by a standard argument of using the Hölder inequality. By interpolating between the $L^{2} \rightarrow L^{2}$ estimate and the $L^{p} \rightarrow L^{q}$ estimates on the line segment $q=3 p^{\prime}$ and $1 \leq p<4$, we obtain that, for $q>\frac{2 n}{n-1}, \frac{n+1}{q}=\frac{n-1}{p^{\prime}}$ and $f \in \mathcal{L}_{1}$,

$$
\left\|(f d \sigma)^{\vee}\right\|_{L_{t, x}^{q}} \lesssim R^{\alpha(R)}\|f\|_{L^{p}(S)}
$$

where $\alpha$ is the step function

$$
\alpha(R)= \begin{cases}-\frac{n-2}{2}\left[1-\frac{2 n}{q(n-1)}\right], & \text { for } R \geq 2 \\ \frac{n-1}{q}, & \text { for } 0<R \leq 1\end{cases}
$$

We remark that the constant above $C R^{\alpha(R)}$ does not depend on $\varepsilon$. By scaling, when $f \in \mathcal{L}_{M}$, under the same conditions on $p$ and $q$,

$$
\left\|(f d \sigma)^{\vee}\right\|_{L_{t, x}^{q}} \lesssim(R M)^{\alpha(R M)}\|f\|_{L^{p}(S)},
$$

where $\alpha$ is defined as above. Then for all cylindrically symmetric $f$ supported on the paraboloid, we decompose it into a sum of dyadically supported functions,

$$
f=\sum_{M: \text { dyadic }} f 1_{\left\{(\tau, \xi): \tau=|\xi|^{2}, M \leq|\xi| \leq 2 M\right\}}=\sum_{M} f_{M}
$$


where $f_{M}:=f 1_{\left\{(\tau, \xi): \tau=|\xi|^{2}, M \leq|\xi| \leq 2 M\right\}}$. Hence

$$
\begin{aligned}
\left\|(f d \sigma)^{\vee}\right\|_{L_{t, x}^{q}\left(\mathbb{R} \times \mathbb{R}^{n-1}\right)} & =\left(\sum_{R}\left\|(f d \sigma)^{\vee}\right\|_{L_{t, x}^{q}\left(\mathbb{R} \times A_{R}\right)}^{q}\right)^{1 / q} \\
& =\left(\sum_{R}\left\|\sum_{M}\left(f_{M} d \sigma\right)^{\vee}\right\|_{L_{t, x}^{q}\left(\mathbb{R} \times A_{R}\right)}^{q}\right)^{1 / q} \\
& \leq\left(\sum_{R}\left(\sum_{M}\left\|\left(f_{M} d \sigma\right)^{\vee}\right\|_{L_{t, x}^{q}\left(\mathbb{R} \times A_{R}\right)}\right)^{q}\right)^{1 / q} \\
& \lesssim\left(\sum_{R}\left(\sum_{M}(R M)^{\alpha(R M)}\left\|f_{M}\right\|_{L^{p}(S)}\right)^{q}\right)^{1 / q} \\
& \lesssim\left(\sum_{M}\left\|f_{M}\right\|_{L^{p}(S)}^{p}\right)^{1 / p}=\|f\|_{L^{p}(S)},
\end{aligned}
$$

where $R>0$ and $M>0$ are both dyadic numbers; in the last inequality, since

$$
\begin{aligned}
& \forall R>0, \sum_{M}(R M)^{\alpha(R M)}<\infty, \\
& \forall M>0, \sum_{R}(R M)^{\alpha(R M)}<\infty,
\end{aligned}
$$

we have used the Schur's test for exponents $p$ and $q$ satisfying the condition $q>\frac{2 n}{n-1}>p \geq 1$.

Remark 2.4. In the cylindrically symmetric case, we remark that $q>\frac{2 n}{n-1}$ is still sharp since it is given by the decay estimate $\left|(d \sigma)^{\vee}(t, \xi)\right| \leq C_{n}(1+$ $|t|+|\xi|)^{(1-n) / 2}$, see e.g., [14, Chapter 8, Theorem 3.1].

Next we state the theorem regarding the bilinear restriction estimates in the cylindrically symmetric case, which is proven in Section 4.

Theorem 2.5. Suppose $f \in \mathcal{L}_{1}$ and $g \in \mathcal{L}_{M}$ with $0<M \leq 1 / 4$. $R>0$ is a dyadic number. Then the following sharp bilinear restriction estimates hold:

- for $q=1$ and $2 \leq p \leq \infty$,

$$
\begin{aligned}
& \left\|\left(f d \sigma_{1}\right)^{\vee}\left(g d \sigma_{2}\right)^{\vee}\right\|_{L_{t, x}^{1}} \\
& \quad \lesssim \min \left\{R M^{\frac{n-2}{2}-\frac{n-1}{p}}, R^{\frac{n}{2}} M^{-1+\frac{n-1}{p^{\prime}}}, R^{n-1} M^{-1+\frac{n-1}{p^{\prime}}}\right\}\|f\|_{L^{p}\left(S_{1}\right)}\|g\|_{L^{p}\left(S_{2}\right)} .
\end{aligned}
$$


- for $q=2$ and $2 \leq p \leq \infty$,

$$
\begin{aligned}
& \left\|\left(f d \sigma_{1}\right)^{\vee}\left(g d \sigma_{2}\right)^{\vee}\right\|_{L_{t, x}^{2}} \\
& \quad \lesssim \min \left\{R^{-\frac{n-2}{2}} M^{\frac{n-1}{2}-\frac{n-1}{p}}, R^{\frac{1}{2}} M^{\frac{n-1}{p^{\prime}}}, R^{\frac{n-1}{2}} M^{\frac{n-1}{p^{\prime}}}\right\}\|f\|_{L^{p}\left(S_{1}\right)}\|g\|_{L^{p}\left(S_{2}\right)} .
\end{aligned}
$$

- for $q=4$ and $4 \leq p \leq \infty, \forall \varepsilon>0$,

$$
\begin{aligned}
& \left\|\left(f d \sigma_{1}\right)^{\vee}\left(g d \sigma_{2}\right)^{\vee}\right\|_{L_{t, x}^{4}} \\
& \lesssim \varepsilon \min \left\{R^{-\frac{3(n-2)}{4}+\varepsilon} M^{\frac{n}{2}-\frac{n-1}{p}}, R^{-\frac{n-2}{4}+\varepsilon} M^{\frac{n-1}{p^{\prime}}}, R^{\frac{n-1}{4}} M^{\frac{n-1}{p^{\prime}}}\right\}\|f\|_{L^{p}\left(S_{1}\right)}\|g\|_{L^{p}\left(S_{2}\right)} .
\end{aligned}
$$

- for $q=3 p^{\prime}$ and $1 \leq p<4$,

$$
\begin{aligned}
& \left\|\left(f d \sigma_{1}\right)^{\vee}\left(g d \sigma_{2}\right)^{\vee}\right\|_{L_{t, x}^{q}} \\
& \lesssim \min \left\{R^{-\frac{n-2}{q^{\prime}}} M^{\frac{n}{2}-\frac{n-1}{p}}, R^{(n-2)\left(\frac{1}{q}-\frac{1}{2}\right)} M^{\frac{n-1}{p^{\prime}}}, R^{\frac{n-1}{q}} M^{\frac{n-1}{p^{\prime}}}\right\}\|f\|_{L^{p}\left(S_{1}\right)}\|g\|_{L^{p}\left(S_{2}\right)} .
\end{aligned}
$$

- for $q=\infty$ and $1 \leq p \leq \infty$,

$$
\begin{aligned}
& \left\|\left(f d \sigma_{1}\right)^{\vee}\left(g d \sigma_{2}\right)^{\vee}\right\|_{L_{t, x}^{\infty}} \\
& \lesssim \min \left\{R^{-(n-2)} M^{\frac{n}{2}-\frac{n-1}{p}}, R^{-\frac{n-2}{2}} M^{\frac{n-1}{p^{\prime}}}, M^{\frac{n-1}{p^{\prime}}}\right\}\|f\|_{L^{p}\left(S_{1}\right)}\|g\|_{L^{p}\left(S_{2}\right)} .
\end{aligned}
$$

where $A$ in $\{A, B, C\}$ is given by the case where $R \geq 1 / M, B$ by $2 \leq R \leq$ $1 / M$ and $C$ by $R \leq 1$. Furthermore, by interpolation we obtain the sharp restriction estimates $L^{p} \times L^{p} \rightarrow L^{q}$ when $p$ and $q$ are in the region determined by these lines (Figure 3).

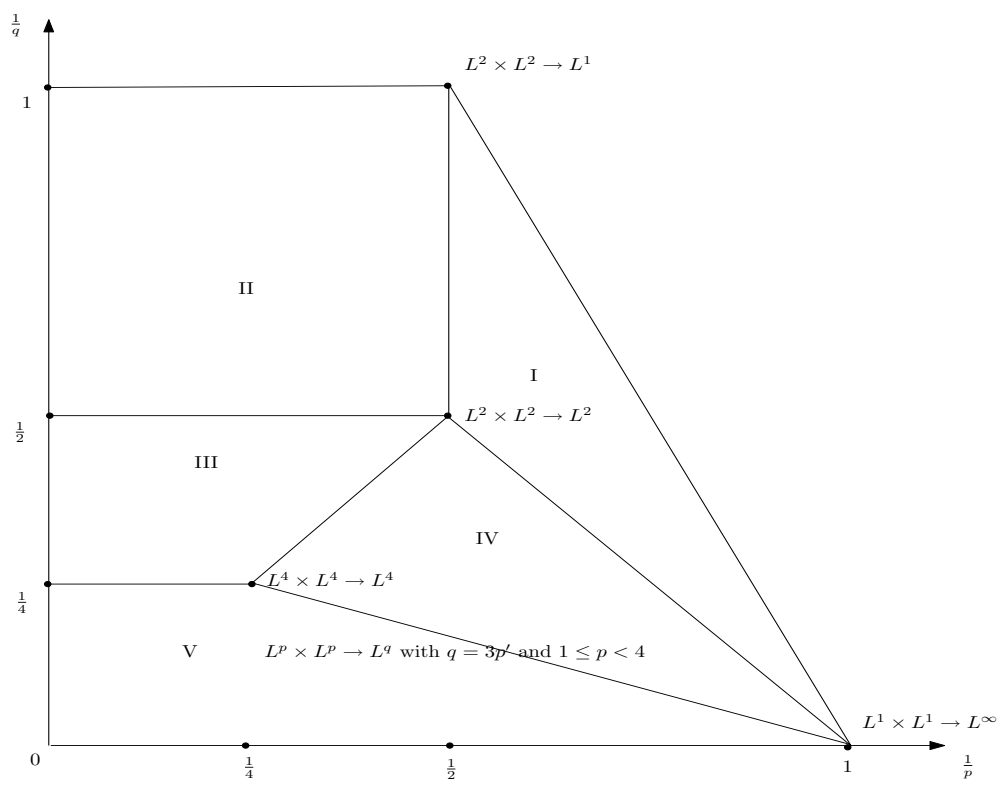

Figure 3: Bilinear restriction estimates on $\mathbb{R} \times A_{R}$. 
Remark 2.6. We observe that $R \geq \frac{1}{M}$ if and only if $A \lesssim B$ and $R \geq 1$ if and only if $B \lesssim C$. In other words, the estimates are "continuous" in the sense that $A \sim B$ when $R \sim 1 / M$ and $B \sim C$ when $R \sim 1$.

As a corollary of Theorem 2.5, we see that the bilinear adjoint restriction conjecture holds for exponents $p$ and $q$ in a larger region.

Corollary 2.7. Suppose $f$ and $g$ are defined as Theorem 2.5. Then the bilinear adjoint restriction conjecture 1.2 holds with constants depending on $p, q, n, S_{1}, S_{2}$ and $M$, whenever $q>\frac{n}{n-1}, \frac{2}{p}+\frac{n}{q}<n$ and $\frac{2}{p}+\frac{1}{q} \leq 2$. These estimates are sharp except for certain endpoints (Figure 4).

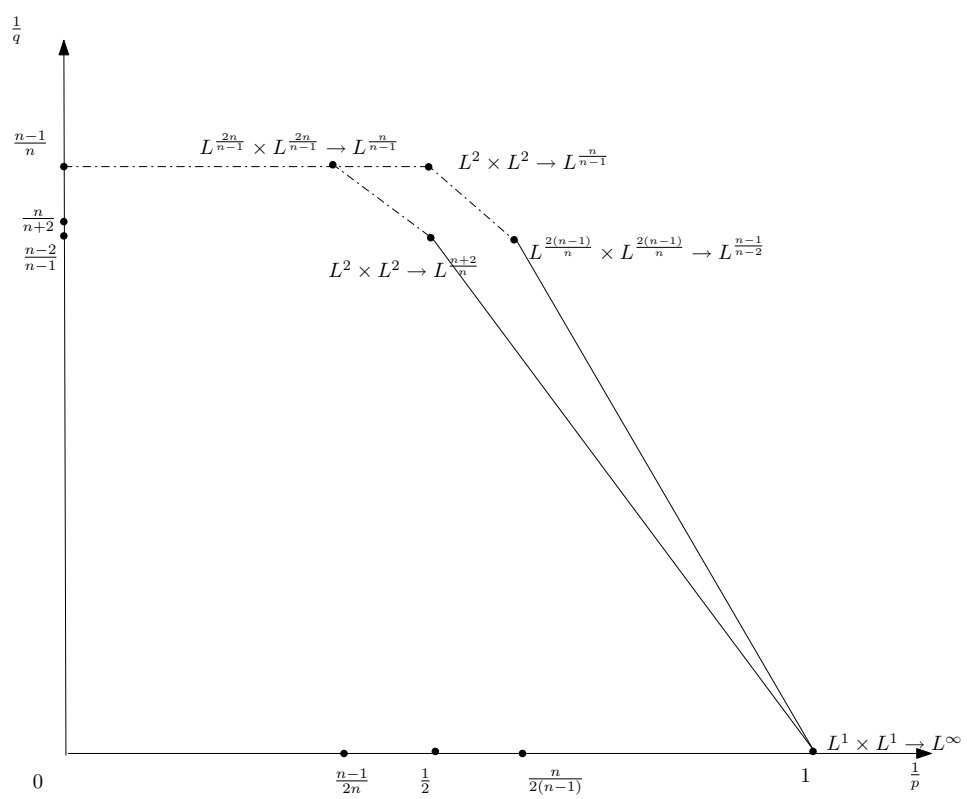

Figure 4: Bilinear restriction estimates for functions in $\mathcal{L}_{1}$ and $\mathcal{L}_{M}$ with $0<M \leq 1 / 4$ (cf. the inside pentagram, the classical range of $p$ and $q$ for Conjecture 1.2).

Remark 2.8. When $S$ is replaced by the lower third of the sphere, the analogous results to Theorems 2.1 and 2.5 hold, which will be accomplished in Section 5. This is essentially due to the common geometric property of non-vanishing Gaussian curvature shared by the sphere and the paraboloid and the fact that the sphere locally resembles the paraboloid, which can be seen from the Taylor expansion $\sqrt{1-|\xi|^{2}} \sim 1-c(|\xi|)|\xi|^{2}$ when $|\xi|$ is small.

Remark 2.9. It is well known that the adjoint restriction estimates are closely related to the Strichartz estimates for the nonlinear dispersive equations such as the Schrödinger equation and the wave equation, see e.g., [16] and [14]. We will establish this connection in our case in Section 6. 
This paper is organized as follows: Section 3 is devoted proving Theorem 2.1 and constructing counterexamples to show the linear estimates are sharp. Section 4 is devoted proving Theorem 2.5 and constructing counterexamples showing the bilinear estimates are sharp. In Section 5 we will establish analogous results for the cylindrically symmetric functions supported on the lower third of the sphere or the cylindrically symmetric and compact hypersurfaces of elliptic type. In Section 6 we will interpret the restriction estimates in terms of solutions to the Schrödinger equation to establish the Strichartz estimates.

\section{Proof of Theorem 2.1: linear estimates and examples}

For any cylindrically symmetric function $f$ on the paraboloid, we set $F(|\xi|)=$ $f\left(|\xi|^{2}, \xi\right)$. We observe that $(f d \sigma)^{\vee}(t, x)$ is also a cylindrically symmetric function. To begin the proof of Theorem 2.1, we first investigate the behavior of $(f d \sigma)^{\vee}$ on $\{|x| \leq 1\}$ via the following proposition.

Proposition 3.1. Suppose $f \in \mathcal{L}_{1}$. Then for any $1 \leq p \leq \infty, q \geq \max \left\{2, p^{\prime}\right\}$ and $R \leq 1$, we have a sharp estimate

$$
\left\|(f d \sigma)^{\vee}\right\|_{L_{t, x}^{q}} \lesssim R^{\frac{n-1}{q}}\|f\|_{L^{p}(S)} .
$$

Proof. If we change to polar coordinates, the left-hand side of (3.1) is

$$
\begin{aligned}
\left\|(f d \sigma)^{\vee}\right\|_{L_{t, x}^{q}} & =\left(\int_{R / 2}^{R} \int_{\mathbb{R}}\left|\int_{1 \leq|\xi| \leq 2} f(\xi) e^{i\left(x \cdot \xi-t \cdot|\xi|^{2}\right)} d \xi\right|^{q} d t d x\right)^{1 / q} \\
& =\left(\int_{R / 2}^{R} \int_{\mathbb{R}}\left|\int_{I} F(s) s^{n-2} e^{-i t s^{2}} \int_{\mathcal{S}^{n-2}} e^{i r s \omega} d \mu(\omega) d s\right|^{q} d t r^{n-2} d r\right)^{1 / q},
\end{aligned}
$$

where $I=[1,2]$. Then we change variables back $s \rightarrow a^{1 / 2}$ to majorize it by

$$
\left(\int_{R / 2}^{R} \int_{\mathbb{R}}\left|\int_{I^{\prime}} a^{(n-3) / 2} F\left(a^{1 / 2}\right)(d \mu)^{\vee}\left(r a^{1 / 2} e_{1}\right) e^{-i t a} d a\right|^{q} d t r^{n-2} d r\right)^{1 / q}
$$

where $I^{\prime}=[1, \sqrt{2}]$ and $e_{1}=(1,0, \ldots, 0) \in \mathbb{R}^{n-1}$.

Then by the Hausdorff-Young inequality when $q>2$ or the Plancherel theorem when $q=2$, changing $s \rightarrow a=s^{2}$ and the fact that $\left\|(d \mu)^{\vee}\right\|_{L_{\omega}^{\infty}} \lesssim 1$, the left-hand side of (3.1) is further bounded by

$$
R^{\frac{n-2}{q}}\left(\int_{R / 2}^{R}\|F\|_{L^{q^{\prime}(I)}}^{q} d r\right)^{1 / q} \sim R^{\frac{n-1}{q}}\|F\|_{L^{q^{\prime}}(I)} .
$$

Then by the Hölder inequality and the fact $\|F\|_{L^{p}(I)} \sim\|f\|_{L^{p}(S)}$, (3.1) follows. 
Now we will construct a counterexample to show the estimate (3.1) is sharp when $1 \leq p \leq \infty$ and $q \geq \max \left\{2, p^{\prime}\right\}$. We take

$$
f\left(|\xi|^{2}, \xi\right)=F(|\xi|)=|\xi|^{-(n-2)} 1_{\{1 \leq|\xi| \leq 2\}} e^{i t_{0}|\xi|^{2}},
$$

where $t_{0} \in \mathbb{R}$. Then the left-hand side of (3.1) reduces to

$$
\left(\int_{R / 2}^{R} \int_{\mathbb{R}}\left|\int_{I} e^{-i\left(t-t_{0}\right) s^{2}} \int_{\mathcal{S}^{n-2}} e^{i r s \omega} d \mu(\omega) d s\right|^{q} d t r^{n-2} d r\right)^{1 / q} .
$$

We choose $r$ and $t$ satisfying that

$$
r \in[R / 100, R / 50],\left|t-t_{0}\right| \leq 1 / 100 .
$$

Then the left-hand side of $(3.1) \gtrsim R^{\frac{n-1}{q}}$ and its right-hand side $\lesssim R^{\frac{n-1}{q}}$. Hence (3.1) is easily seen to be sharp.

Before investigating the behavior of $(f d \sigma)^{\vee}$ on $|x| \geq 1$, we shall exploit the spatial rotation-invariant symmetry of $f$ in the following proposition.

Lemma 3.2 (Fourier-Bessel formula). Suppose $f$ is a cylindrically symmetric function supported on the paraboloid. Then

$$
\begin{aligned}
& (f d \sigma)^{\vee}(t, x) \\
& =c_{n} r^{-\frac{n-2}{2}} \int_{I} F(s) s^{\frac{n-2}{2}} e^{i\left(r s-t s^{2}\right)} d s+c_{n} r^{-\frac{n-2}{2}} \int_{I} F(s) s^{\frac{n-2}{2}} e^{-i\left(r s+t s^{2}\right)} d s \\
& \quad+c_{n} \int_{I} F(s) s^{n-2} e^{-i t s^{2}-i r s} \int_{0}^{\infty} e^{-r s y} y^{\frac{n-4}{2}}\left[(y+2 i)^{\frac{n-4}{2}}-(2 i)^{\frac{n-4}{2}}\right] d y d s
\end{aligned}
$$

$$
-c_{n} \int_{I} F(s) s^{n-2} e^{-i t s^{2}+i r s} \int_{0}^{\infty} e^{-r s y} y^{\frac{n-4}{2}}\left[(y-2 i)^{\frac{n-4}{2}}-(-2 i)^{\frac{n-4}{2}}\right] d y d s,
$$

where $r=|x|$ and $I$ is the projection interval in the radial direction.

Proof. We first expand $(f d \sigma)^{\vee}$ in the polar coordinates,

$$
(f d \sigma)^{\vee}(t, x)=\int_{\{|\xi| \in I\}} f\left(|\xi|^{2}, \xi\right) e^{i\left(x \xi-t|\xi|^{2}\right)} d \xi=\int_{I} F(s) e^{-i t s^{2}} s^{n-2}(d \mu)^{\vee}\left(r s e_{1}\right) d s,
$$

where $d \mu$ is the surface measure of the sphere $\mathcal{S}^{n-2}$.

On the one hand, the inverse Fourier transform of $d \mu$ is given by

$$
(d \mu)^{\vee}(\xi)=c_{n}|\xi|^{\frac{3-n}{2}} J_{\frac{n-3}{2}}(|\xi|),
$$

where $J_{\frac{n-3}{2}}$ is the Bessel function of order $\frac{n-3}{2}$, see e.g. [14] or [15]. 
On the other hand, by using the same argument as proving [15, Lemma 3.11], we obtain that, for fixed $m \geq 0$,

$$
\begin{aligned}
J_{m}(r)= & c_{m} \frac{e^{i r}-e^{-i r}}{r^{1 / 2}}+c_{m} r^{m} e^{-i r} \int_{0}^{\infty} e^{-r y} y^{\frac{2 m-1}{2}}\left[(y+2 i)^{\frac{2 m-1}{2}}-(2 i)^{\frac{2 m-1}{2}}\right] d y \\
& -c_{m} r^{m} e^{i r} \int_{0}^{\infty} e^{-r y} y^{\frac{2 m-1}{2}}\left[(y-2 i)^{\frac{2 m-1}{2}}-(-2 i)^{\frac{2 m-1}{2}}\right] d y .
\end{aligned}
$$

Hence (3.2) holds after we combine these two estimates and set $m=\frac{n-3}{2}$.

Therefore we define the error term of $(f d \sigma)^{\vee}$ by

$$
\begin{aligned}
\mathcal{E} f(t, x):= & \pm c_{n} \int_{I} F(s) s^{n-2} e^{-i t s^{2} \mp i r s} \times \\
& \times \int_{0}^{\infty} e^{-r s y} y^{\frac{n-4}{2}}\left[(y \pm 2 i)^{\frac{n-4}{2}}-( \pm 2 i)^{\frac{n-4}{2}}\right] d y d s,
\end{aligned}
$$

where by \pm we denote a sum of two terms where + and - appear alternatively. Heuristically, one should think of $\mathcal{E} f$ as a term comparable to $r^{-n / 2} \int_{I} F(s) s^{\frac{n-4}{2}} e^{-i t s^{2}} d s$, which comes from estimating the error term of Bessel function $J_{m}(r)$ by $r^{-3 / 2}$. At the first approximation, this simplification is easy to deal with and intuitively provides what the bound involving the error term should be. However in this paper we will establish it rigorously in the following proposition, which shows that the information about its contribution to the linear estimates when $|x| \geq 1$. It is acceptable compared to the main term estimates established in the next propositions.

Proposition 3.3. Suppose $f \in \mathcal{L}_{1}$. Then for any $q \geq \max \left\{2, p^{\prime}\right\}$ and any dyadic number $R \geq 2$ and $f \in L^{p}(S)$,

$$
\|\mathcal{E} f\|_{L_{t, x}^{q}} \lesssim R^{-\frac{n}{2}+\frac{n-1}{q}}\|f\|_{L^{p}(S)}
$$

Proof. We set

$$
E(r):=\int_{0}^{\infty} e^{-r y} y^{\frac{n-4}{2}}\left[(y \pm 2 i)^{\frac{n-4}{2}}-( \pm 2 i)^{\frac{n-4}{2}}\right] d y .
$$

For $r \geq 1$, we first estimate $E(r)$ by repeating the proof of [15, Lemma 3.11] for readers' convenience.

$$
\begin{aligned}
|E(r)| \leq & \int_{0}^{1} e^{-r y} y^{\frac{n-4}{2}}\left|(y \pm 2 i)^{\frac{n-4}{2}}-( \pm 2 i)^{\frac{n-4}{2}}\right| d y+ \\
& +\int_{1}^{\infty} e^{-r y} y^{\frac{n-4}{2}}\left|(y+2 i)^{\frac{n-4}{2}}-(2 i)^{\frac{n-4}{2}}\right| d y \\
=: & I+I I
\end{aligned}
$$


For $I$, where $0 \leq y \leq 1$, by the mean value theorem we have $\mid(y \pm 2 i)^{\frac{n-4}{2}}-$ $( \pm 2 i)^{\frac{n-4}{2}} \mid \lesssim y$. For $I I$, where $y \geq 1$, we take $y$ out and then use the mean value theorem to obtain $\left|(y \pm 2 i)^{\frac{n-4}{2}}-( \pm 2 i)^{\frac{n-4}{2}}\right| \lesssim y^{\frac{n-4}{2}}$. Then combining these estimates above,

$$
\begin{aligned}
|E(r)| & \leq \int_{0}^{1} e^{-r y} y^{\frac{n-2}{2}} d y+\int_{1}^{\infty} e^{-r y} y^{n-4} d y \\
& \lesssim r^{-n / 2} \int_{0}^{r} e^{-y} y^{\frac{n}{2}-1} d y+r^{-(n-3)} \int_{r}^{\infty} e^{-y} y^{n-4} d y .
\end{aligned}
$$

By the definition of the Gamma function $\Gamma$,

$$
r^{-n / 2} \int_{0}^{r} e^{-y} y^{\frac{n}{2}-1} d y \lesssim \Gamma(n / 2) r^{-n / 2}
$$

Then using integration by parts $n-4$ times when $n \geq 4$ and $y^{-1} \leq r^{-1}$ when $n=3$, we obtain $r^{-(n-3)} \int_{r}^{\infty} e^{-y} y^{n-4} d y \lesssim e^{-r} r^{-1}$.

Since $e^{-r} r^{-1+\frac{n}{2}}$ is continuous on $[1, \infty)$ and decays to 0 as $r \rightarrow \infty$, $e^{-r} r^{-1} \lesssim r^{-\frac{n}{2}}$ holds for $r \geq 1$. Therefore

$$
|E(r)| \lesssim r^{-n / 2}
$$

Next let us turn to the estimate (3.3). By changing to polar coordinates, the left-hand side of (3.3) is comparable to

$$
\left(\int_{R / 2}^{R} \int_{\mathbb{R}}\left|\int_{I} F(s) s^{n-2} e^{-i t s^{2} \mp i r s} E(r s) d s\right|^{q} d t r^{n-2} d r\right)^{1 / q}
$$

where $I=[1,2]$. After changing variables $s=a^{1 / 2},(3.5)$ is comparable to

$$
\left(\int_{R / 2}^{R} \int_{\mathbb{R}}\left|\int_{I^{\prime}} F\left(a^{1 / 2}\right) a^{\frac{n-3}{2}} E\left(r a^{1 / 2}\right) e^{\mp i r a^{1 / 2}} e^{-i t a} d a\right|^{q} d t r^{n-2} d r\right)^{1 / q},
$$

where $I^{\prime}=[1, \sqrt{2}]$. Then by the Hausdorff-Young inequality when $q>2$ or the Plancherel theorem when $q=2$, changing variables back $a=s^{2}$ and $s \sim 1$, the left-hand side of (3.5) is further majorized by

$$
\left(\left.\left.\int_{R / 2}^{R} \int_{\mathbb{R}}\left|\int_{I}\right| F(s) E(r s)\right|^{q^{\prime}} d s\right|^{q / q^{\prime}} d t r^{n-2} d r\right)^{1 / q}
$$

Since $r s \geq 1$ for any $r \in[R / 2, R]$ and $s \in I,(3.4)$ and the Hölder inequality give $R^{-\frac{n}{2}+\frac{n-1}{q}}\|F\|_{L^{p}(I)}$. Since $\|F\|_{L^{p}(I)} \sim\|f\|_{L^{p}(S)}$, (3.3) follows. 
When $|x| \geq 1$, we are left with estimating the main term of $(f d \sigma)^{\vee}$,

$$
\mathcal{M} f(t, x):=c_{n} r^{-\frac{n-2}{2}} \int_{I} F(s) s^{\frac{n-2}{2}} e^{i\left( \pm r s-t s^{2}\right)} d s
$$

where by \pm we denote the summation of two terms. We call it the heuristic approximation of $(f d \sigma)^{\vee}$. We are going to prove the positive part "estimates" of Theorem 2.1 in the following four propositions. In the remainder of this section, we will prove its negative part "sharpness" by certain counterexamples.

Proposition 3.4 ( $q=2$ line). Suppose $f \in \mathcal{L}_{1}$. Then for $q=2,2 \leq p \leq \infty$ and $R \geq 2$, we have a sharp estimate

$$
\left\|(f d \sigma)^{\vee}\right\|_{L_{t, x}^{2}} \lesssim R^{1 / 2}\|f\|_{L^{p}(S)} .
$$

Proof. We observe that it is sufficient to estimate the term of $\mathcal{M} f$ with + sign. In the propositions followed, we will make the same reduction unless specified. Hence by the heuristic approximation $(3.6)$ of $(f d \sigma)^{\vee}$ with + sign, changing variables and then the Plancherel theorem in $t$ followed by the Hölder inequality,

$$
\begin{aligned}
\left\|(f d \sigma)^{\vee}\right\|_{L_{t, x}^{2}} & =\left(\int_{R / 2 \leq|x| \leq R} \int_{\mathbb{R}}\left|(f d \sigma)^{\vee}(t, x)\right|^{2} d t d x\right)^{1 / 2} \\
& \sim\left(\int_{R / 2}^{R} \int_{\mathbb{R}}\left|r^{-\frac{n-2}{2}} \int_{I} F(s) s^{\frac{n-2}{2}} e^{i\left[r s-t s^{2}\right]} d s\right|^{2} d t r^{n-2} d r\right)^{1 / 2} \\
& =\left(\int_{R / 2}^{R} \int_{\mathbb{R}}\left|\int_{I} F(s) s^{\frac{n-2}{2}} e^{i r s} e^{-t s^{2}} d s\right|^{2} d t d r\right)^{1 / 2} \\
& =\left(\int_{R / 2}^{R}\|F\|_{L_{s}^{2}(I)}^{2} d r\right)^{1 / 2} \lesssim R^{1 / 2}\|f\|_{L^{2}(S)} \lesssim R^{1 / 2}\|f\|_{L^{p}(S)},
\end{aligned}
$$

where $I=[1,2], 2 \leq p \leq \infty$. Hence (3.7) follows.

Now let us deal with the estimates on the line $q=4$. The estimate $L^{4} \rightarrow L^{4}$ is the endpoint of two dimensional $(n=2)$ linear adjoint restriction conjecture and hence the classical $T T^{*}$ approach, namely the Carleson-Sjölin argument used in Proposition 3.6, unfortunately fails because we can not apply the Hardy-Littlewood-Sobolev inequality at one step. Instead, we can perform a Whitney-type decomposition to $I$ to create some frequency separation. Similar arguments can be found in [20], [22], [23]. 
Proposition $3.5\left(q=4\right.$ line). Suppose $f \in \mathcal{L}_{1}$. Then for $q=4,4 \leq p \leq \infty$ and $\varepsilon>0$ and $R \geq 2$, we have a sharp estimate up to $R^{\varepsilon}$,

$$
\left\|(f d \sigma)^{\vee}\right\|_{L_{t, x}^{4}} \lesssim \varepsilon R^{-(n-2) / 4+\varepsilon}\|f\|_{L^{p}(S)} .
$$

Proof. By the same reasoning as that used in the proof of Proposition 3.4, we will only prove the estimate when $p=4$. By the heuristic approximation $(3.6)$ of $(f d \sigma)^{\vee}$ with + sign,

$$
\left\|(f d \sigma)^{\vee}\right\|_{L_{t, x}^{4}}^{2} \sim R^{-(n-2) / 2}\left(\int_{R / 2}^{R} \int_{\mathbb{R}}\left|\int_{I} F(s) s^{\frac{n-2}{2}} e^{i\left(r s-t s^{2}\right)} d s\right|^{4} d t d r\right)^{1 / 2},
$$

where $I=[1,2]$.

We set $(F d \sigma)^{\vee}(t, r):=\int_{I} F(s) s^{\frac{n-2}{2}} e^{i\left[r s-t s^{2}\right]} d s$, which can be regarded as the inverse space-time Fourier transform of $f(\tau, \xi)|\xi|^{\frac{n-2}{2}}$ restricted to the parabola. To prove (3.8), it suffices to prove that, for any $\varepsilon>0$,

$$
\left(\int_{R / 2}^{R} \int_{\mathbb{R}}\left|(F d \sigma)^{\vee}(F d \sigma)^{\vee}\right|^{2} d t d r\right)^{1 / 2} \lesssim_{\varepsilon} R^{\varepsilon}\|F\|_{L^{4}}^{2}
$$

Next we will perform a Whitney-type decomposition to $I=[1,2]$. For each $j \geq 0$ we break up $I$ into $O\left(2^{j}\right)$ dyadic intervals $\tau_{k}^{j}$ of length $2^{-j}$, and write $\tau_{k}^{j} \simeq \tau_{k^{\prime}}^{j}$ if $\tau_{k}^{j}$ and $\tau_{k^{\prime}}^{j}$ are not adjacent but have adjacent parents. For each $j \geq 0$, let $F=\sum F_{k}^{j}$ where $F_{k}^{j}=F 1_{\tau_{k}^{j}}$. Then

$$
(F d \sigma)^{\vee}(F d \sigma)^{\vee}=\sum_{j} \sum_{k, k^{\prime}: \tau_{k}^{j} \simeq \tau_{k^{\prime}}^{j}}\left(F_{k}^{j} d \sigma\right)^{\vee}\left(F_{k^{\prime}}^{j} d \sigma\right)^{\vee}
$$

From the triangle inequality, the left-hand side of (3.9) is bounded by

$$
\begin{aligned}
\sum_{2^{j} \leq R} & \left\|\sum_{k, k^{\prime}: \tau_{k}^{j} \simeq \tau_{k^{\prime}}^{j}}\left(F_{k}^{j} d \sigma\right)^{\vee}\left(F_{k^{\prime}}^{j} d \sigma\right)^{\vee}\right\|_{L_{t, r}^{2}(\mathbb{R} \times \mathbb{R})}+ \\
& +\sum_{2^{j} \geq R} \sum_{k, k^{\prime}: \tau_{k}^{j} \simeq \tau_{k^{\prime}}^{j}}\left\|\left(F_{k}^{j} d \sigma\right)^{\vee}\left(F_{k^{\prime}}^{j} d \sigma\right)^{\vee}\right\|_{L_{t, r}^{2}\left(\mathbb{R} \times A_{R}\right)}=: A+B .
\end{aligned}
$$

We will first estimate $A$. By the quasi-orthogonality property of functions among $\left(F_{k}^{j} d \sigma\right)^{\vee}\left(F_{k^{\prime}}^{j} d \sigma\right)^{\vee}[23$, Lemma 6.1],

$$
A \lesssim \sum_{2^{j} \leq R}\left(\sum_{k, k^{\prime}: \tau_{k}^{j} \simeq \tau_{k^{\prime}}^{j}}\left\|\left(F_{k}^{j} d \sigma\right)^{\vee}\left(F_{k^{\prime}}^{j} d \sigma\right)^{\vee}\right\|_{L_{t, r}^{2}(\mathbb{R} \times \mathbb{R})}^{2}\right)^{1 / 2}
$$


By using the Plancherel theorem and the Cauchy-Schwarz inequality and $s_{i} \sim 1$ for $i=1,2$,

$$
\left\|\left(F_{k}^{j} d \sigma\right)^{\vee}\left(F_{k^{\prime}}^{j} d \sigma\right)^{\vee}\right\|_{L_{t, r}^{2}(\mathbb{R} \times \mathbb{R})}^{2} \lesssim\left\|F_{k}^{j}\right\|_{L^{2}\left(I_{k}^{j}\right)}^{2}\left\|F_{k^{\prime}}^{j}\right\|_{L^{2}\left(I_{k^{\prime}}^{j}\right)}^{2}\left\|d \sigma_{k}^{j} * d \sigma_{k^{\prime}}^{j}\right\|_{L^{\infty}}
$$

where $d \sigma_{k}^{j}$ and $d \sigma_{k^{\prime}}^{j}$ are two arc measures of the parabola $\left\{\tau=|\xi|^{2}\right\}$ in $\mathbb{R} \times \mathbb{R}$ supported on $\tau_{k}^{j}$ and $\tau_{k^{\prime}}^{j}$, respectively.

On the one hand, from the geometric properties of the paraboloid,

$$
\left\|d \sigma_{k}^{j} * d \sigma_{k^{\prime}}^{j}\right\|_{L^{\infty}} \lesssim 2^{j} .
$$

On the other hand, by the Hölder inequality, $\left\|F_{k}^{j}\right\|_{L^{2}\left(I_{k}^{j}\right)} \leq 2^{-j / 4}\left\|F_{k}^{j}\right\|_{L^{4}\left(I_{k}^{j}\right)}$. Thus after combining these estimates,

$$
A \lesssim \sum_{2^{j} \leq R}\left(\sum_{k, k^{\prime}: \tau_{k}^{j} \simeq \tau_{k^{\prime}}^{j}}\left\|F_{k}^{j}\right\|_{L^{4}\left(I_{k}^{j}\right)}^{2}\left\|F_{k^{\prime}}^{j}\right\|_{L^{4}\left(I_{k^{\prime}}^{j}\right)}^{2}\right)^{1 / 2}
$$

We also observe that for each $k$, there are $O(1) k^{\prime}$ such that $\tau_{k}^{j} \simeq \tau_{k^{\prime}}^{j}$. Hence by the Cauchy-Schwarz inequality, for any $\varepsilon>0$,

$$
A \lesssim(\log R)\|F\|_{L^{4}}^{2} \lesssim \varepsilon R^{\varepsilon}\|F\|_{L^{4}}^{2}
$$

Next let us estimate $B$. On the one hand, by the Cauchy-Schwarz inequality,

$$
\left\|\left(F_{k^{\prime}}^{j} d \sigma\right)^{\vee}\right\|_{L_{t, x}^{\infty}} \lesssim 2^{-j / 2}\left\|F_{k^{\prime}}^{j}\right\|_{L^{2}\left(\tau_{k^{\prime}}^{j}\right.} .
$$

On the other hand, by the Plancherel theorem in $t$,

$$
\left\|\left(F_{k}^{j} d \sigma\right)^{\vee}\right\|_{L_{t, r}^{2}\left(\mathbb{R} \times A_{R}\right)}=\left(\int_{R / 2}^{R} \int_{\mathbb{R}}\left|\left(F_{k}^{j} d \sigma\right)^{\vee}\right|^{2} d t d r\right)^{1 / 2} \lesssim R^{1 / 2}\left\|F_{k}^{j}\right\|_{L^{2}\left(\tau_{k}^{j}\right)} .
$$

Since there are $O(1) k^{\prime}$ such that $\tau_{k}^{j} \simeq \tau_{k^{\prime}}^{j}$ for each $k$, by using the CauchySchwarz inequality,

$$
B \lesssim R^{1 / 2} \sum_{2^{j} \geq R} \sum_{k, k^{\prime}: \tau_{k}^{j} \simeq \tau_{k^{\prime}}^{j}} 2^{-j / 2}\left\|F_{k^{\prime}}^{j}\right\|_{L^{2}\left(\tau_{k^{\prime}}^{j}\right)}\left\|F_{k}^{j}\right\|_{L^{2}\left(\tau_{k}^{j}\right)} \lesssim R^{1 / 2} \sum_{2^{j} \geq R} 2^{-j / 2}\|F\|_{L^{2}}^{2} .
$$

Thus summing in $j$ and using the Hölder inequality, (3.9) follows.

In contrast to the proof of the estimate $L^{4} \rightarrow L^{4}$ in Proposition 3.5, the estimates $L^{p} \rightarrow L^{q}$ when $q=3 p^{\prime}$ and $1 \leq p<4$ can be proven by the Carleson-Sjölin argument or equivalently the $T T^{*}$ method. Such arguments can also be used to prove the non-endpoint Strichartz estimates as in [8]. 
Proposition 3.6 ( $q=3 p^{\prime}$ line). Suppose $f \in \mathcal{L}_{1}$. Then for $1 \leq p<4$, $q=3 p^{\prime}$ and $R \geq 2$, we have a sharp estimate

$$
\left\|(f d \sigma)^{\vee}\right\|_{L_{t, x}^{q}} \lesssim R^{(n-2)(1 / q-1 / 2)}\|f\|_{L^{p}(S)} .
$$

Proof. By the heuristic approximation (3.6) with + sign,

$$
\left\|(f d \sigma)^{\vee}\right\|_{L_{t, x}^{q}} \sim R^{(n-2)\left(\frac{1}{q}-\frac{1}{2}\right)}\left(\int_{R / 2}^{R} \int_{\mathbb{R}}\left|\int_{I} F(s) s^{\frac{n-2}{2}} e^{i\left(r s-t s^{2}\right)} d s\right|^{q} d t d r\right)^{1 / q},
$$

where $I=[1,2]$.

Setting $(F d \sigma)^{\vee}(t, r):=\int_{I} F(s) s^{\frac{n-2}{2}} e^{i\left(r s-t s^{2}\right)} d s$, we see that it suffices to prove

$$
\left(\int_{R / 2}^{R} \int_{\mathbb{R}}\left|\int_{I} F(s) s^{\frac{n-2}{2}} e^{i\left(r s-t s^{2}\right)} d s\right|^{q} d t d r\right)^{1 / q} \lesssim\|F\|_{L^{p}(S)} .
$$

Squaring $(F d \sigma)^{\vee}$, we obtain

$$
\left\{(F d \sigma)^{\vee}(t, r)\right\}^{2}=\int_{I \times I} F\left(s_{1}\right) F\left(s_{2}\right)\left(s_{1} s_{2}\right)^{\frac{n-2}{2}} e^{i\left(r \cdot\left(s_{1}+s_{2}\right)-t\left(s_{1}^{2}+s_{2}^{2}\right)\right)} d s_{1} d s_{2},
$$

which is an oscillatory integral with a phase function $r\left(s_{1}+s_{2}\right)-t\left(s_{1}^{2}+s_{2}^{2}\right)$. Its Hessian is $2\left(s_{2}-s_{1}\right)$ which vanishes when $s_{1}=s_{2}$. But we can make a change of variables $\left(s_{1}, s_{2}\right) \rightarrow(a, b)$ with $a=s_{1}+s_{2}, b=s_{1}^{2}+s_{2}^{2}$. It is easy to see that the Jacobian is $2\left(s_{2}-s_{1}\right)$. Let $\Omega$ be the image in $\mathbb{R} \times \mathbb{R}$ of $I \times I$ under such change of variables. Then $\left\{(F d \sigma)^{\vee}(t, r)\right\}^{2}=\int_{\Omega} \tilde{F}(a, b) e^{i(r a-t b)} d a d b$, where $\tilde{F}(a, b)=F\left(s_{1}\right) F\left(s_{2}\right)\left(s_{1} s_{2}\right)^{\frac{n-2}{2}} /\left|s_{1}-s_{2}\right|$ is a function of $s_{1}$ and $s_{2}$.

Setting $q=2 r^{\prime}$. Since $r^{\prime}>2$, by the Hausdorff-Young inequality and $s_{i} \sim 1$ for $i=1,2$,

$$
\begin{aligned}
\left\|(F d \sigma)^{\vee}(t, r)\right\|_{L^{q}\left(\mathbb{R} \times A_{R}\right)}^{2} & \leq\left\|\left\{(F d \sigma)^{\vee}(t, r)\right\}^{2}\right\|_{L^{r^{\prime}}(\mathbb{R} \times \mathbb{R})} \\
& \leq\left(\int_{\Omega}\left|\tilde{F}\left(s_{1}, s_{2}\right)\right|^{r} d s_{1} d s_{2}\right)^{1 / r} \\
& \sim\left(\int_{I^{2}}\left|F\left(s_{1}\right)\right|^{r}\left|F\left(s_{2}\right)\right|^{r} \frac{1}{\left|s_{1}-s_{2}\right|^{r-1}} d s_{1} d s_{2}\right)^{1 / r} \\
& =\left(\int_{I}\left|F\left(s_{1}\right)\right|^{r} \int_{I}\left|F\left(s_{2}\right)\right|^{r} \frac{1}{\left|s_{1}-s_{2}\right|^{1-(2-r)}} d s_{2} d s_{1}\right)^{1 / r} \\
& \sim\left(\int_{I}\left|F\left(s_{1}\right)\right|^{r} I_{2-r}\left(|F|^{r}\right)\left(s_{1}\right) d s_{1}\right)^{1 / r},
\end{aligned}
$$


where $I_{2-r}$ is the Riesz potential of order $2-r$ defined via the spatial Fourier transform by $\widehat{I_{s} f}(\xi)=|\xi|^{-s} \hat{f}(\xi)$. Since $\|f\|_{L^{p}} \sim\|F\|_{L^{p}(I)}$, it then suffices to prove that

$$
\left(\int_{I}|F|^{r} I_{2-r}\left(|F|^{r}\right) d s_{1}\right)^{1 / r} \lesssim\|F\|_{L^{p}(I)}^{2}
$$

By the Hölder inequality, we obtain

$$
\int_{I}|F|^{r} I_{2-r}\left(|F|^{r}\right) d s_{1} \leq\left\||F|^{r}\right\|_{L^{p / r}(I)}\left\|I_{2-r}\left(|F|^{r}\right)\right\|_{L^{1 /(1-r / p)}(I)} .
$$

Since $\left\||F|^{r}\right\|_{L^{p / r}(I)}^{1 / r}=\|F\|_{L^{p}(I)}$, it suffices to show $\left\|I_{2-r}\left(|F|^{r}\right)\right\|_{L^{1 /(1-r / p)}} \lesssim$ $\left\||F|^{r}\right\|_{L^{p / r}(I)}$, which will follow from the Hardy-Littlewood-Sobolev inequality. Hence the inequality (3.11) follows.

Proposition 3.7 ( $q=\infty$ line). Suppose $f \in \mathcal{L}_{1}$. Then for $q=\infty, 1 \leq p \leq \infty$ and $R \geq 2$, we have a sharp estimate

$$
\left\|(f d \sigma)^{\vee}\right\|_{L_{t, x}^{\infty}} \lesssim R^{-(n-2) / 2}\|f\|_{L^{p}(S)} .
$$

Proof. By the heuristic approximation (3.6) of $(f d \sigma)^{\vee}$ with + sign and the Hölder inequality, for any $p \geq 1$,

$$
\left\|(f d \sigma)^{\vee}\right\|_{L_{t, x}^{\infty}} \sim R^{-(n-2) / 2}\left\|\int_{I} F(s) s^{\frac{n-2}{2}} e^{i\left(r s-t s^{2}\right)} d s\right\|_{L_{t, r}^{\infty}} \lesssim R^{-(n-2) / 2}\|f\|_{L^{p}(S)} .
$$

Now we see that the restriction estimates in Theorem 2.1 follow from Propositions 3.3, 3.4, 3.5, 3.6 and 3.7.

The remainder of this section is devoted constructing counterexamples. In view of the propositions above, we will use the main term of $(f d \sigma)^{\vee}$,

$$
\mathcal{M} f(t, x)=c_{n} r^{-\frac{n-2}{2}} \int_{I} F(s) s^{\frac{n-2}{2}} e^{i\left( \pm r s-t s^{2}\right)} d s
$$

since the bound $B$ given by the error terms are much smaller than that by the main terms when $R$ is sufficiently large .

Our first counterexample is of Knapp-type, which is designed to show the estimates in the region $I$ in Figure 1 determined by the estimates $L^{2} \rightarrow L^{2}$, $L^{4} \rightarrow L^{4}$ and $L^{1} \rightarrow L^{\infty}$ are sharp. The strength of the standard Knapp example or its variants lie in the idea of using both spatial localization and frequency localization. In this example, we will only show that the estimate $L^{2} \rightarrow L^{2}$ is sharp since the computations for others are similar. 
Example $3.8(\mathbf{I})$. If $R \geq 2$, the $L^{2} \rightarrow L^{2}$ estimate goes back to (3.7) in Proposition 3.4. We take

$$
f\left(|\xi|^{2}, \xi\right)=F(|\xi|)=|\xi|^{-(n-2) / 2} 1_{\left\{1 \leq|\xi| \leq 1+R^{-1 / 2}\right\}} e^{-i r_{0}|\xi|+i t_{0}|\xi|^{2}}
$$

where $r_{0} \in[R / 2, R]$ and $t_{0} \in \mathbb{R}$. Thus the left-hand side of (3.7) is comparable to

$$
\left(\int_{R / 2}^{R} \int_{\mathbb{R}}\left|\int_{1}^{1+R^{-1 / 2}} e^{\left[-i\left(t-t_{0}\right) s^{2}+i\left( \pm r-r_{0}\right) s\right]} d s\right|^{2} d t d r\right)^{1 / 2}
$$

where by \pm it denotes a summation of two terms. We observe that

$$
\begin{aligned}
& \left|\int_{1}^{1+R^{-1 / 2}} e^{\left[-i\left(t-t_{0}\right) s^{2}+i\left( \pm r-r_{0}\right) s\right]} d s\right| \\
& \quad=\left|\int_{1}^{1+R^{-1 / 2}} e^{-i\left\{\left(t-t_{0}\right)(s-1)^{2}-\left[\left( \pm r-r_{0}\right)-2\left(t-t_{0}\right)\right](s-1)\right\}} d s\right|
\end{aligned}
$$

and hence choose $r \in[R / 2, R]$ and $t \in \mathbb{R}$ such that

$$
\begin{aligned}
R / 100 \leq t-t_{0} & \leq R / 50 \\
\left.\mid\left(r-r_{0}\right)-2\left(t-t_{0}\right)\right](s-1) \mid & \leq R^{1 / 2} / 100
\end{aligned}
$$

Thus $r$ and $t$ are in the intersection region of two tubes whose size is of $R^{1 / 2} \times R$.

With this choice of $r$ and $t,\left|\left(t-t_{0}\right)(s-1)^{2}-\left[\left(r-r_{0}\right)-2\left(t-t_{0}\right)\right](s-1)\right|$ is less than a small number, say $\pi / 6$. Then by direct computations, the term with + sign will be bounded below by $R^{1 / 4}$. However for the term with sign, given this choice of $r$ and $t$, we see that the roots of the quadratic polynomial, $\left(t-t_{0}\right)(s-1)^{2}-\left[\left(r+r_{0}\right)+2\left(t-t_{0}\right)\right](s-1)$, will be strictly less than -1 , which consequently are not located in the interval $\left[0, R^{1 / 2}\right]$. Thus by the principle of non-stationary phase, we see that the term with sign will be bounded above by $O_{N}\left(R^{-N}\right)$ for any $N>0$. Then by choosing $N$ sufficiently large, from the triangle inequality the left-hand side of (3.7) $\gtrsim R^{1 / 4}$. Also it is easy to see that its right-hand side $\lesssim R^{1 / 4}$. Thus we see that the estimate $L^{2} \rightarrow L^{2}$ when $R \geq 2$ is sharp.

Our second counterexample is to show that the estimates in the region II in Figure 1 determined by the lines $q=2$ and $q=4$ are sharp. In this example we will only show the estimates on the line $q=2$ in Proposition 3.4 are sharp by using the principle of stationary phase. 
Example 3.9 (II). If $R \geq 2$, the estimate $L^{p} \rightarrow L^{2}$ when $2 \leq p \leq \infty$ goes back to Proposition 3.4. We take the example

$$
f\left(|\xi|^{2}, \xi\right)=F(|\xi|)=|\xi|^{-\frac{n-2}{2}} 1_{\{1 \leq|\xi| \leq 2\}} e^{-i r_{0}|\xi|+i t_{0}|\xi|^{2}},
$$

where $r_{0} \in[R / 2, R]$ and $t_{0} \in \mathbb{R}$. Then the left-hand side of (3.7) is comparable to

$$
\left(\int_{R / 2}^{R} \int_{\mathbb{R}}\left|\int_{I} e^{-i\left\{\left(t-t_{0}\right)(s-1)^{2}-\left[\left( \pm r-r_{0}\right)-2\left(t-t_{0}\right)\right](s-1)\right\}} d s\right|^{2} d t d r\right)^{1 / 2} .
$$

We choose $r \in[R / 100, R / 50]$ and $t \in \mathbb{R}$ such that $\left(r-r_{0}\right) / 2\left(t-t_{0}\right) \in[1,2]$. Then

$$
-\frac{-\left[\left(r-r_{0}\right)-2\left(t-t_{0}\right)\right]}{2\left(t-t_{0}\right)} \in[0,1],-\frac{\left(r+r_{0}\right)+2\left(t-t_{0}\right)}{2\left(t-t_{0}\right)}<-1 .
$$

Then from the principles of stationary phase and non-stationary phase,

$$
\begin{aligned}
& \left|\int_{I} e^{-i\left\{\left(t-t_{0}\right)(s-1)^{2}-\left[\left(r-r_{0}\right)-2\left(t-t_{0}\right)\right](s-1)\right\}} d s\right| \gtrsim R^{-1 / 2}, \\
& \left|\int_{I} e^{-i\left\{\left(t-t_{0}\right)(s-1)^{2}+\left[\left(r+r_{0}\right)+2\left(t-t_{0}\right)\right](s-1)\right\}} d s\right| \lesssim_{N} R^{-N},
\end{aligned}
$$

for any $N \geq 0$. Then if choosing $N$ sufficiently large, the triangle inequality gives

$$
\left(\int_{R / 2}^{R} \int_{\mathbb{R}}\left|\int_{I} e^{-i\left\{\left(t-t_{0}\right)(s-1)^{2}-\left[\left( \pm r-r_{0}\right)-2\left(t-t_{0}\right)\right](s-1)\right\}} d s\right|^{2} d t d r\right)^{1 / 2} \gtrsim R^{1 / 2} .
$$

Its right-hand side $\lesssim R^{1 / 2}$ for $2 \leq p \leq \infty$. Thus we see that the estimates on the line $q=2$ when $R \geq 2$ are sharp.

The third counterexample shows that the estimates inside the region III determined by lines $q=4, q=3 p^{\prime}$ and $q=\infty$ in Figure 1 are sharp. In this example, we will only carry out the computations for the estimates $L^{p} \rightarrow L^{q}$ on the line $q=\infty$ in Proposition 3.7.

Example 3.10 (III). If $R \geq 2$, the estimate $L^{p} \rightarrow L^{\infty}$ when $1 \leq p \leq \infty$ goes back to Proposition 3.7. We take

$$
f\left(|\xi|^{2}, \xi\right)=F(|\xi|)=|\xi|^{-\frac{n-2}{2}} 1_{\{1 \leq|\xi| \leq 2\}} e^{-i r_{0}|\xi|+i t_{0}|\xi|^{2}},
$$

where $r_{0} \in[R / 2, R]$ and $t_{0} \in \mathbb{R}$. They are chosen such that $\left\|(f d \sigma)^{\vee}\right\|_{L_{t, x}^{\infty}}$ can be realized at $\left(t_{0}, x_{0}\right)$ with $r_{0}=\left|x_{0}\right|$. 
Hence the left-hand side of the inequality (3.12) is comparable to

$$
R^{-\frac{n-2}{2}}\left|\int_{I} e^{i\left( \pm r_{0}-r_{0}\right) s} d s\right|
$$

Since $r_{0} \in[R / 2, R],\left|\int_{I} e^{-i 2 r_{0} s} d s\right| \lesssim R^{-N}$ holds for any $N \geq 0$. Then the triangle inequality yields $R^{-\frac{n-2}{2}}\left|\int_{I} e^{i\left( \pm r_{0}-r_{0}\right) s} d s\right| \gtrsim R^{-\frac{n-2}{2}}$. On the other hand, the right-hand side of $(3.12) \lesssim R^{-(n-2) / 2}$ for $1 \leq p \leq \infty$. Hence the estimates on the line $q=\infty$ when $R \geq 2$ are sharp.

For lines $q=4$ and $q=3 p^{\prime}$, the estimates go back to (3.8) and (3.10). We choose $r \in[R / 2, R]$ and $t$ such that $2 \leq r-r_{0} \leq 4,2 \leq t-t_{0} \leq 4$. Then by the same reasoning as Example 3.8, these estimates are sharp.

Thus the proof of Theorem 2.1 is complete.

\section{Proof of Theorem 2.5: bilinear estimates and exam- ples}

For $f \in \mathcal{L}_{1}$ and $g \in \mathcal{L}_{M}$ with $0<M \leq 1 / 4$, we set $I_{1}=[1,2], I_{M}=[M, 2 M]$ and $F(|\xi|)=f\left(|\xi|^{2}, \xi\right), G(|\xi|)=g\left(|\xi|^{2}, \xi\right)$. In the bilinear case, 1 and $1 / M$ will bring in two natural separation scales in the physical space. In light of the proof of Theorem 2.1, we will have the following permutations of the product $\left(f d \sigma_{1}\right)^{\vee}\left(g d \sigma_{2}\right)^{\vee}$.

- when $|x|=r \geq 1 / M$,

$$
\left|\left(f d \sigma_{1}\right)^{\vee}\left(g d \sigma_{2}\right)^{\vee}\right|=|\mathcal{M} f \mathcal{M} g+\mathcal{M} f \mathcal{E} g+\mathcal{M} g \mathcal{E} f+\mathcal{E} f \mathcal{E} g| .
$$

- when $1 \leq|x|=r \leq 1 / M$,

$$
\left|\left(f d \sigma_{1}\right)^{\vee}\left(g d \sigma_{2}\right)^{\vee}\right|=\left|\mathcal{M} f\left(g d \sigma_{2}\right)^{\vee}+\mathcal{E} f\left(g d \sigma_{2}\right)^{\vee}\right| .
$$

- when $|x| \leq 1,\left|\left(f d \sigma_{1}\right)^{\vee}\left(g d \sigma_{2}\right)^{\vee}\right|$ remains unchanged.

We are going to prove the "estimates" part of Theorem 2.5 via the following three propositions and its "sharpness" part by building counterexamples in three cases followed.

Proposition 4.1. Suppose $f \in \mathcal{L}_{1}$ and $g \in \mathcal{L}_{M}$ with $0<M \leq 1 / 4$, and $R \leq 1$ is a dyadic number. Then we have sharp estimates

- for $q=1$ and $2 \leq p \leq \infty$,

$$
\left\|\left(f d \sigma_{1}\right)^{\vee}\left(g d \sigma_{2}\right)^{\vee}\right\|_{L_{t, x}^{1}} \lesssim R^{n-1} M^{-1+\frac{n-1}{p^{\prime}}}\|f\|_{L^{p}\left(S_{1}\right)}\|g\|_{L^{p}\left(S_{2}\right)} .
$$

- for $q \geq \max \left\{2, p^{\prime}\right\}$,

$$
\left\|\left(f d \sigma_{1}\right)^{\vee}\left(g d \sigma_{2}\right)^{\vee}\right\|_{L_{t, x}^{q}} \lesssim R^{\frac{n-1}{q}} M^{\frac{n-1}{p^{\prime}}}\|f\|_{L^{p}\left(S_{1}\right)}\|g\|_{L^{p}\left(S_{2}\right)} .
$$


Proof. If we change to the polar coordinates, the left-hand side of (4.1) reduces to

$$
\begin{aligned}
\int_{R / 2}^{R} \int_{\mathbb{R}} & \mid \int_{I_{1}} F\left(s_{1}\right) e^{-i t s_{1}^{2}}(d \mu)^{\vee}\left(r s_{1} e_{1}\right) s_{1}^{n-2} d s_{1} \times \\
& \times \int_{I_{M}} G\left(s_{2}\right) e^{-i t s_{2}^{2}}(d \mu)^{\vee}\left(r s_{2} e_{1}\right) s_{2}^{n-2} d s_{2} \mid d t r^{n-2} d r .
\end{aligned}
$$

We use the Cauchy-Schwarz inequality and the Plancherel theorem in $t$ to bound (4.3) by

$$
R^{n-1} M^{n-2}\|F\|_{L^{2}\left(I_{1}\right)} M^{-1 / 2}\|G\|_{L^{2}\left(I_{M}\right)} .
$$

Then by the Hölder inequality, (4.4) is bounded by

$$
R^{n-1} M^{n-2-\frac{n-1}{p}}\|f\|_{L^{p}\left(S_{1}\right)}\|g\|_{L^{p}\left(S_{2}\right)} .
$$

Hence the inequality (4.1) follows.

To prove (4.2), by the Hölder inequality,

$$
\left\|\left(f d \sigma_{1}\right)^{\vee}\left(g d \sigma_{2}\right)^{\vee}\right\|_{L_{t, x}^{q}} \lesssim\left\|\left(f d \sigma_{1}\right)^{\vee}\right\|_{L_{t, x}^{q}}\left\|\left(g d \sigma_{2}\right)^{\vee}\right\|_{L_{t, x}^{\infty}} .
$$

On the one hand, by Proposition 3.1,

$$
\left\|\left(f d \sigma_{1}\right)^{\vee}\right\|_{L_{t, x}^{q}} \lesssim R^{\frac{n-1}{q}}\|f\|_{L^{p}\left(S_{1}\right)} .
$$

On the other hand, by the Hölder inequality,

$$
\left\|\left(g d \sigma_{2}\right)^{\vee}\right\|_{L_{t, x}^{\infty}} \lesssim M^{\frac{n-1}{p^{\prime}}}\|g\|_{L^{p}\left(S_{2}\right)} .
$$

Hence the inequality (4.2) follows.

The following proposition concerns the case where $1 \leq|x| \leq 1 / M$.

Proposition 4.2. Suppose $f \in \mathcal{L}_{1}$ and $g \in \mathcal{L}_{M}$ with $0<M \leq 1 / 4$, and $2 \leq R \leq 1 / M$. Then

- for $q=1$ and $2 \leq p \leq \infty$,

$$
\left\|\left(f d \sigma_{1}\right)^{\vee}\left(g d \sigma_{2}\right)^{\vee}\right\|_{L_{t, x}^{1}} \lesssim R^{\frac{n}{2}} M^{-1+\frac{n-1}{p^{\prime}}}\|f\|_{L^{p}\left(S_{1}\right)}\|g\|_{L^{p}\left(S_{2}\right)} .
$$

- for $q \geq \max \left\{2, p^{\prime}\right\}$,

$$
\left\|\left(f d \sigma_{1}\right)^{\vee}\left(g d \sigma_{2}\right)^{\vee}\right\|_{L_{t, x}^{q}} \lesssim\left\|R^{*}\right\|_{L^{p} \rightarrow L^{q}} M^{\frac{n-1}{p^{\prime}}}\|f\|_{L^{p}\left(S_{1}\right)}\|g\|_{L^{p}\left(S_{2}\right)},
$$

where $\left\|R^{*}\right\|_{L^{p} \rightarrow L^{q}}$ denotes the operator norm of $f \rightarrow(f d \sigma)^{\vee}$ from $L^{p}\left(S_{1}\right)$ to $L_{t, x}^{q}$ given by Theorem 2.1 . 
Proof. To prove (4.5), it suffices to prove the following inequalities by Lemma 3.2,

$\int_{R / 2}^{R} \int_{\mathbb{R}}\left|\mathcal{M} f\left(t, r e_{1}\right)\right|\left|\left(g d \sigma_{2}\right)^{\vee}\left(t, r e_{1}\right)\right| d t r^{n-2} d r \lesssim R^{\frac{n}{2}} M^{-1+\frac{n-1}{p^{\prime}}}\|f\|_{L^{p}\left(S_{1}\right)}\|g\|_{L^{p}\left(S_{2}\right)}$,

and

$\int_{R / 2}^{R} \int_{\mathbb{R}}\left|\mathcal{E} f\left(t, r e_{1}\right)\right|\left|\left(g d \sigma_{2}\right)^{\vee}\left(t, r e_{1}\right)\right| d t r^{n-2} d r \lesssim R^{\frac{n}{2}-1} M^{-1+\frac{n-1}{p^{\prime}}}\|f\|_{L^{p}\left(S_{1}\right)}\|g\|_{L^{p}\left(S_{2}\right)}$.

These two estimates above can be proven along similar lines as proving (4.1). We choose to prove the second. The Hölder inequality yields,

$$
\left\|\left(f d \sigma_{1}\right)^{\vee}\left(g d \sigma_{2}\right)^{\vee}\right\|_{L_{t, x}^{q}} \lesssim\left\|\left(f d \sigma_{1}\right)^{\vee}\right\|_{L_{t, x}^{q}}\left\|\left(g d \sigma_{2}\right)^{\vee}\right\|_{L_{t, x}^{\infty}} .
$$

Then by using the same reasoning as in the proof of (4.2), the inequality (4.6) follows. We note that the error term $\mathcal{E} f$ gives a better decay estimate as expected.

Next let us concentrate on the case where $|x| \geq 1 / M$. As indicated at the beginning of this section, we will have to deal with estimates involving $|\mathcal{M} f \mathcal{M} g|,|\mathcal{M} f \mathcal{E} g|,|\mathcal{E} f \mathcal{M} g|$ and $|\mathcal{E} f \mathcal{E} g|$.

Proposition 4.3 (Bilinear main term estimates). Suppose $f \in \mathcal{L}_{1}$ and $g \in \mathcal{L}_{M}$ with $0<M \leq 1 / 4$, and $R \geq 1 / M$. Then

- for $q=1$ and $2 \leq p \leq \infty$,

$$
\left\|\left(f d \sigma_{1}\right)^{\vee}\left(g d \sigma_{2}\right)^{\vee}\right\|_{L_{t, x}^{1}} \lesssim R M^{\frac{n-2}{2}-\frac{n-1}{p}}\|f\|_{L^{p}\left(S_{1}\right)}\|g\|_{L^{p}\left(S_{2}\right)} .
$$

- for $q=2$ and $2 \leq p \leq \infty$,

$$
\left\|\left(f d \sigma_{1}\right)^{\vee}\left(g d \sigma_{2}\right)^{\vee}\right\|_{L_{t, x}^{2}} \lesssim R^{-\frac{n-2}{2}} M^{\frac{n-1}{2}-\frac{n-1}{p}}\|f\|_{L^{p}\left(S_{1}\right)}\|g\|_{L^{p}\left(S_{2}\right)} .
$$

- for $q \geq \max \left\{4,3 p^{\prime}\right\}$ and $1 \leq p \leq \infty$,

$$
\left\|\left(f d \sigma_{1}\right)^{\vee}\left(g d \sigma_{2}\right)^{\vee}\right\|_{L_{t, x}^{q}} \lesssim\left\|R^{*}\right\|_{L^{p} \rightarrow L^{q}} R^{-\frac{n-2}{2}} M^{\frac{n}{2}-\frac{n-1}{p}}\|f\|_{L^{p}\left(S_{1}\right)}\|g\|_{L^{p}\left(S_{2}\right)} .
$$

Proof. To prove (4.7), it suffices to prove the following inequalities

$$
\begin{aligned}
&\|\mathcal{M} f \mathcal{M} g\|_{L_{t, x}^{1}} \lesssim R M^{\frac{n-2}{2}-\frac{n-1}{p}}\|f\|_{L^{p}\left(S_{1}\right)}\|g\|_{L^{p}\left(S_{2}\right)} . \\
&\|\mathcal{E} f \mathcal{E} g\|_{L_{t, x}^{1}} \lesssim R^{-1} M^{\frac{n-4}{2}-\frac{n-1}{p}}\|f\|_{L^{p}\left(S_{1}\right)}\|g\|_{L^{p}\left(S_{2}\right)} . \\
&\|\mathcal{M} f \mathcal{E} g\|_{L_{t, x}^{1}} \lesssim M^{\frac{n-4}{2}-\frac{n-1}{p}}\|f\|_{L^{p}\left(S_{1}\right)}\|g\|_{L^{p}\left(S_{2}\right)} . \\
&\|\mathcal{E} f \mathcal{M} g\|_{L_{t, x}^{1}} \lesssim M^{\frac{n-2}{2}-\frac{n-1}{p}}\|f\|_{L^{p}\left(S_{1}\right)}\|g\|_{L^{p}\left(S_{2}\right)} .
\end{aligned}
$$


In what follows we will only prove (4.10) since other estimates involving error terms will follow similarly. In fact these inequalities give better decay estimates than those given by (4.10). By the heuristic approximation (3.6) with + sign, the left-hand side of (4.10) reduces to

$$
\int_{R / 2}^{R} \int_{\mathbb{R}}\left|\int_{I_{1}} F\left(s_{1}\right) s_{1}^{\frac{n-2}{2}} e^{i\left(r s_{1}-t s_{1}^{2}\right)} d s_{1} \int_{I_{M}} G\left(s_{2}\right) s_{2}^{\frac{n-2}{2}} e^{i\left(r s_{2}-t s_{2}^{2}\right)}\right| d t d r .
$$

After changing variables and using the Cauchy-Schwarz inequality and the Plancherel theorem in $t$, we see that (4.11) is bounded by

$$
R M^{\frac{n-3}{2}}\|F\|_{L^{2}\left(I_{1}\right)}\|G\|_{L^{2}\left(I_{M}\right)} \sim R M^{-\frac{1}{2}}\|f\|_{L^{2}\left(S_{1}\right)}\|g\|_{L^{2}\left(S_{2}\right)} .
$$

Then from the Hölder inequality, the inequality (4.10) follows. Similarly, to prove (4.8), it suffices to prove the following inequalities

$$
\begin{aligned}
&\|\mathcal{M} f \mathcal{M} g\|_{L_{t, x}^{2}} \lesssim R^{-\frac{n-2}{2}} M^{\frac{n-1}{2}-\frac{n-1}{p}}\|f\|_{L^{p}\left(S_{1}\right)}\|g\|_{L^{p}\left(S_{2}\right)} . \\
&\|\mathcal{E} f \mathcal{E} g\|_{L_{t, x}^{2}} \lesssim R^{-\frac{n+1}{2}} M^{\frac{n-2}{2}-\frac{n-1}{p}}\|f\|_{L^{p}\left(S_{1}\right)}\|g\|_{L^{p}\left(S_{2}\right)} . \\
&\|\mathcal{M} f \mathcal{E} g\|_{L_{t, x}^{2}} \lesssim R^{-\frac{n}{2}+\frac{1}{2}} M^{\frac{n-2}{2}-\frac{n-1}{p}}\|f\|_{L^{p}\left(S_{1}\right)}\|g\|_{L^{p}\left(S_{2}\right)} . \\
&\|\mathcal{E} f \mathcal{M} g\|_{L_{t, x}^{2}} \lesssim R^{-\frac{n}{2}+\frac{1}{2}} M^{\frac{n}{2}-\frac{n-1}{p}}\|f\|_{L^{p}\left(S_{1}\right)}\|g\|_{L^{p}\left(S_{2}\right)} .
\end{aligned}
$$

Since the estimates above involving error terms give better decay estimates than (4.12), we will also only prove (4.12). We rewrite its left-hand side as

$$
\begin{aligned}
& R^{-\frac{n-2}{2}}\left(\int_{R / 2}^{R} \int_{\mathbb{R}} \mid \int_{I_{1} \times I_{M}} F\left(s_{1}\right) G\left(s_{2}\right) \times\right. \\
& \left.\quad \times\left.\left(s_{1} s_{2}\right)^{\frac{n-2}{2}} e^{i\left(r\left(s_{1}+s_{2}\right)-t\left(s_{1}^{2}+s_{2}^{2}\right)\right.} d s_{1} d s_{2}\right|^{2} d t d r\right)^{1 / 2} .
\end{aligned}
$$

Setting $x:=s_{1}+s_{2}$ and $y:=s_{1}^{2}+s_{2}^{2}$, we observe that the Jacobian $\sim$ $|1-M| \sim 1$ provided $M \leq 1 / 4$. From the Plancherel theorem both in $t$ and $r$, the left-hand side of (4.12) is further majorized by

$$
R^{-\frac{n-2}{2}} M^{\frac{n-2}{2}}\|F\|_{L^{2}\left(I_{1}\right)}\|G\|_{L^{2}\left(I_{M}\right)} \sim R^{-\frac{n-2}{2}}\|f\|_{L^{2}\left(S_{1}\right)}\|g\|_{L^{2}\left(S_{2}\right)} .
$$

By using the Hölder inequality again, we see that (4.12) follows. Finally we prove (4.9). In fact, it suffices to prove the following two inequalities

$$
\begin{aligned}
\left\|\left(f d \sigma_{1}\right)^{\vee} \mathcal{M} g\right\|_{L_{t, x}^{q}} & \lesssim\left\|R^{*}\right\|_{L^{p} \rightarrow L^{q}} R^{-\frac{n-2}{2}} M^{\frac{n}{2}-\frac{n-1}{p}}\|f\|_{L^{p}\left(S_{1}\right)}\|g\|_{L^{p}\left(S_{2}\right)} . \\
\left\|\left(f d \sigma_{1}\right)^{\vee} \mathcal{E} g\right\|_{L_{t, x}^{q}} & \lesssim\left\|R^{*}\right\|_{L^{p} \rightarrow L^{q}} R^{-\frac{n}{2}} M^{\frac{n}{2}-1-\frac{n-1}{p}}\|f\|_{L^{p}\left(S_{1}\right)}\|g\|_{L^{p}\left(S_{2}\right)} .
\end{aligned}
$$

The first follows from the Hölder inequality and the linear estimate in Theorem 2.1, and the second follows along similar lines. 
Therefore the restriction estimates in Theorem 2.5 are obtained from Propositions 4.1, 4.2 and 4.3.

In the remainder of this section we will construct counterexamples to show these estimates are sharp or nearly sharp up to $R^{\varepsilon}$. Since the error terms give much better decay estimates, we will use the heuristic approximations (3.6) of $\left(f d \sigma_{1}\right)^{\vee}$ and $\left(g d \sigma_{2}\right)^{\vee}$ when computing these examples. We will distinguish them into three cases as follows.

Case 1: $R \geq 1 / M$.

We start with a common example to show the estimates in the region $I$ in Figure 3 determined by $L^{2} \times L^{2} \rightarrow L^{1}, L^{2} \times L^{2} \rightarrow L^{2}$ and $L^{1} \times L^{1} \rightarrow L^{\infty}$ are sharp by using the idea coming from standard Knapp example. In the following example, we will only do the computations when $p=2$ and $q=1$.

Example $4.4(\mathbf{I})$. If $R \geq 1 / M, R M^{-\frac{1}{2}}$ is best possible in the following inequality

$$
\left\|\left(f d \sigma_{1}\right)^{\vee}\left(g d \sigma_{2}\right)^{\vee}\right\|_{L_{t, x}^{1}} \lesssim R M^{-\frac{1}{2}}\|f\|_{L^{2}\left(S_{1}\right)}\|g\|_{L^{2}\left(S_{2}\right)} .
$$

We take

$$
\begin{aligned}
& f\left(|\xi|^{2}, \xi\right)=F(|\xi|)=|\xi|^{-\frac{n-2}{2}} e^{i\left(-r_{0}|\xi|+t_{0}|\xi|^{2}\right)} 1_{\left\{1 \leq|\xi| \leq 1+R^{-1} M\right\}} \\
& g\left(|\eta|^{2}, \eta\right)=G(|\eta|)=|\eta|^{-\frac{n-2}{2}} e^{i\left(-r_{0}|\eta|+t_{0}|\eta|^{2}\right)} 1_{\left\{M \leq|\eta| \leq M+R^{-1}\right\}}
\end{aligned}
$$

where $r_{0} \in[R / 2, R]$ and $t_{0} \in \mathbb{R}$. By the heuristic approximation (3.6), the left-hand side of (4.13) is comparable to

$$
\int_{R / 2}^{R} \int_{\mathbb{R}}\left|\int_{1}^{1+R^{-1} M} e^{i\left[\left( \pm r-r_{0}\right) s_{1}-\left(t-t_{0}\right) s_{1}^{2}\right]} d s_{1} \int_{M}^{M+R^{-1}} e^{i\left[\left( \pm r-r_{0}\right) s_{2}-\left(t-t_{0}\right) s_{2}^{2}\right]} d s_{2}\right| d t d r
$$

which we understood is a summation of four terms. For the integral on $\left[1,1+R^{-1} M\right]$, we will choose $r$ and $t$ such that $R / 100 \leq r-r_{0} \leq R / 50$ and $R M^{-1} / 100 \leq t-t_{0} \leq R M^{-1} / 50$. Then we have

$$
\begin{aligned}
& \left|\left[\left(r-r_{0}\right)-2\left(t-t_{0}\right)\right]\left(s_{1}-1\right)-\left(t-t_{0}\right)\left(s_{1}-1\right)^{2}\right| \leq 1, \\
& \quad-\frac{\left(r+r_{0}\right)+2\left(t-t_{0}\right)}{2\left(t-t_{0}\right)}=-\left(1+\frac{r-r_{0}}{2\left(t-t_{0}\right)}\right) \notin\left[0, R^{-1} M\right] .
\end{aligned}
$$

Hence the integral on $\left[1,1+R^{-1} M\right]$ with $+\operatorname{sign}$ is $\gtrsim R^{-1} M$ while the one with $-\operatorname{sign} \lesssim_{N} R^{-N}$ for any $N \geq 0$. Similarly for the integral on 
$\left[M, M+R^{-1}\right]$ with this choice of $r$ and $t$. Then if $N$ is sufficiently large, the triangle inequality gives

$$
\begin{aligned}
\int_{R / 2}^{R} \int_{\mathbb{R}} \mid \int_{1}^{1+R^{-1} M} & e^{i\left[\left( \pm r-r_{0}\right) s_{1}-\left(t-t_{0}\right) s_{1}^{2}\right]} d s_{1} \\
& \times \int_{M-R^{-1}}^{M} e^{i\left[\left( \pm r-r_{0}\right) s_{2}-\left(t-t_{0}\right) s_{2}^{2}\right]} d s_{2} \mid d t d r \gtrsim 1 .
\end{aligned}
$$

Then by direct computations, the right-hand side of $(4.13) \lesssim 1$. Thus we see that the estimate $L^{2} \times L^{2} \rightarrow L^{1}$ is sharp when $R \geq 1 / M$.

By modifying the above "narrow" Example 4.4, namely taking a linear combination to create a "spreading-out" example, we will show that the estimates in the region $I I$ in Figure 3 determined by the lines $q=1$ and $q=2$ are sharp by using the Khintchine inequality. A similar construction by Lee and Vargas can be found in [10] to show the sharp null form estimates for the wave equation. In the following example we will only do computations for the estimates on the line $q=1$.

Example 4.5 (II). If $R \geq 1 / M, R M^{(n-2) / 2-(n-1) / p}$ is best possible in the following inequality

$$
\left\|\left(f d \sigma_{1}\right)^{\vee}\left(g d \sigma_{2}\right)^{\vee}\right\|_{L_{t, x}^{1}} \lesssim R M^{\frac{n-2}{2}-\frac{n-1}{p}}\|f\|_{L^{p}\left(S_{1}\right)}\|g\|_{L^{p}\left(S_{2}\right)},
$$

where $2 \leq p \leq \infty$.

We define two index sets $J:=\left\{j \in \mathbb{Z}: 1 \leq j \leq\left[R M^{-1}\right]\right\}$ and $K:=\{k \in$ $\mathbb{Z}: 1 \leq k \leq[R M]\}$, where $[x]$ denotes the biggest integer which is less than or equal to $x \in \mathbb{R}$. For each $j \in J, k \in K$ we define

$$
\begin{aligned}
& f_{j}\left(|\xi|^{2}, \xi\right)=F_{j}(|\xi|)=|\xi|^{\frac{n-2}{2}} e^{i\left(-r_{0}|\xi|+t_{0}|\xi|^{2}\right)} 1_{\left\{1+(j-1) R^{-1} M \leq|\xi| \leq 1+j R^{-1} M\right\}} \\
& g_{k}\left(|\eta|^{2}, \eta\right)=G_{k}(|\eta|)=|\eta|^{-\frac{n-2}{2}} e^{i\left(-r_{0}|\eta|+t_{0}|\eta|^{2}\right)} 1_{\left\{M+(k-1) R^{-1} \leq|\eta| \leq M+k R^{-1}\right\}}
\end{aligned}
$$

Also we set $f=\sum_{j \in J} \varepsilon_{j} f_{j}$ and $g=\sum_{k \in K} \tilde{\varepsilon}_{k} g_{k}$, where $\left\{\varepsilon_{j}: j \in J\right\}$ and $\left\{\tilde{\varepsilon}_{k}: k \in K\right\}$ are sets of i.i.d. (independent identically distributed) random variables taking \pm 1 with an equal probability $1 / 2$. Note that $f_{j}$ and $g_{k}$ are "narrow", disjoint and in the form of Example 4.4; but $f$ and $g$ "spread out" and support on the whole set $S_{1}$ and $S_{2}$. By the Khintchine inequality, we estimate the left-hand side of (4.14) by

$$
\mathbb{E}\left(\left\|\left(f d \sigma_{1}\right)^{\vee}\left(g d \sigma_{2}\right)^{\vee}\right\|_{L_{t, x}^{1}}\right) \sim\left\|\left(\sum_{j, k}\left|\left(f_{j} d \sigma_{1}\right)^{\vee}\left(g_{k} d \sigma_{2}\right)^{\vee}\right|^{2}\right)^{1 / 2}\right\|_{L_{t, x}^{1}},
$$


where $\mathbb{E}(X)$ denotes the expectation of the random variable $X$. By the heuristic approximation (3.6), the right-hand side of (4.15) is comparable to

$$
\begin{aligned}
& \int_{R / 2}^{R} \int_{\mathbb{R}}\left(\sum_{j, k} \mid \int_{a_{j}-R^{-1} M}^{a_{j}} F_{j}\left(s_{1}\right) e^{i\left[\left( \pm r-r_{0}\right) s_{1}-\left(t-t_{0}\right) s_{1}^{2}\right]} d s_{1} \times\right. \\
& \left.\times\left.\int_{b_{k}-R^{-1}}^{b_{k}} G_{k}\left(s_{2}\right) e^{i\left[\left( \pm r-r_{0}\right) s_{2}-\left(t-t_{0}\right) s_{2}^{2}\right]} d s_{2}\right|^{2}\right)^{1 / 2} d t d r,
\end{aligned}
$$

where $a_{j}=1+j R^{-1} M$ and $b_{k}=M+k R^{-1}$, and by \pm we denote a summation of four terms. We choose $r$ and $t$ such that $R / 100 \leq r-r_{0} \leq R / 50$ and $R M^{-1} \leq t-t_{0} \leq R M^{-1} / 50$. By this choice of $r$ and $t$ and similar discussions as in Example 4.4, the triangle inequality gives,

$$
\begin{aligned}
\mid \int_{a_{j}-R^{-1} M}^{a_{j}} F_{j}\left(s_{1}\right) e^{i\left[\left( \pm r-r_{0}\right) s_{1}-\left(t-t_{0}\right) s_{1}^{2}\right]} d s_{1} \times \\
\quad \times \int_{b_{k}-R^{-1}}^{b_{k}} G_{k}\left(s_{2}\right) e^{i\left[\left( \pm r-r_{0}\right) s_{2}-\left(t-t_{0}\right) s_{2}^{2}\right]} d s_{2} \mid \gtrsim R^{-2} M .
\end{aligned}
$$

Then (4.16) is bounded below by $R^{2} M^{-1}(|J||K|)^{1 / 2} R^{-2} M$, i.e., $(|J||K|)^{1 / 2}$. Here $|J| \sim R M^{-1}$ denotes the cardinality of the index set $J$, similarly for $|K| \sim M R$. Hence we obtain that the left-hand side of (4.14) is $\gtrsim R$. On the other hand, the right-hand side of $(4.14) \lesssim R$ for $2 \leq p \leq \infty$. Hence the estimates on the line $q=1$ when $R \geq 1 / M$ are sharp.

The following example shows that the estimates in the region III in Figure 3 determined by the lines $q=2$ and $q=4$ are sharp by the principle of stationary phase. We do computations when $q=2$.

Example 4.6 (III). If $R \geq 1 / M, R^{-\frac{n-2}{2}} M^{\frac{n-1}{2}-\frac{n-1}{p}}$ is best possible in the following inequality.

$$
\left\|\left(f d \sigma_{1}\right)^{\vee}\left(g d \sigma_{2}\right)^{\vee}\right\|_{L_{t, x}^{2}} \lesssim R^{-\frac{n-2}{2}} M^{\frac{n-1}{2}-\frac{n-1}{p}}\|f\|_{L^{p}\left(S_{1}\right)}\|g\|_{L^{p}\left(S_{2}\right)},
$$

where $2 \leq p \leq \infty$.

We take

$$
\begin{aligned}
& f\left(|\xi|^{2}, \xi\right)=|\xi|^{-\frac{n-2}{2}} e^{i\left(-r_{0}|\xi|+t_{0}|\xi|^{2}\right)} 1_{\{1 \leq|\xi| \leq 2\}}, \\
& g\left(|\eta|^{2}, \eta\right)=|\eta|^{-\frac{n-2}{2}} e^{i\left(-r_{0}|\eta|+t_{0}|\eta|^{2}\right)} 1_{\{M \leq|\eta| \leq 2 M\}},
\end{aligned}
$$

where $r_{0} \in[R / 2, R]$ and $t_{0} \in \mathbb{R}$. By the heuristic approximation (3.6), the left-hand side of (4.17) is comparable to

$$
R^{-\frac{n-2}{2}}\left(\int_{R / 2}^{R} \int_{\mathbb{R}}\left|\int_{I_{1}} e^{i\left[\left( \pm r-r_{0}\right) s_{1}-\left(t-t_{0}\right) s_{1}^{2}\right]} d s_{1} \int_{I_{M}} e^{i\left[\left( \pm r-r_{0}\right) s_{2}-\left(t-t_{0}\right) s_{2}^{2}\right]} d s_{2}\right|^{2} d t d r\right)^{1 / 2} .
$$


We choose $r \in[R / 2, R]$ and $t$ such that $M^{-1} / 100 \leq r-r_{0} \leq M^{-1} / 50$ and $\left(r-r_{0}\right) / 2\left(t-t_{0}\right) \in I_{1}$. Then from the principles of stationary phase and non-stationary phase, for any $N \geq 0$,

$$
\begin{aligned}
\left|\int_{I_{1}} e^{i\left[\left(r-r_{0}\right) s_{1}-\left(t-t_{0}\right) s_{1}^{2}\right]} d s_{1}\right| & \gtrsim M^{1 / 2}, \\
\left|\int_{I_{1}} e^{-i\left[\left(r+r_{0}\right) s_{1}+\left(t-t_{0}\right) s_{1}^{2}\right]} d s_{1}\right| & \lesssim_{N} M^{N}, \\
\left|\int_{s_{2} \sim M} e^{i\left[\left(r-r_{0}\right) s_{2}-\left(t-t_{0}\right) s_{2}^{2}\right]} d s_{2}\right| & \gtrsim M, \\
\left|\int_{s_{2} \sim M} e^{-i\left[\left(r+r_{0}\right) s_{2}+\left(t-t_{0}\right) s_{2}^{2}\right]} d s_{2}\right| & \lesssim_{N} M^{N} .
\end{aligned}
$$

Then from the triangle inequality, the left-hand side of $(4.17) \gtrsim R^{-\frac{(n-2)}{2}} M^{1 / 2}$. By direct computations, the right-hand side of $(4.17) \lesssim R^{-\frac{(n-2)}{2}} M^{1 / 2}$. Thus the estimates on the line $q=2$ when $R \geq 1 / M$ are sharp.

The next example will show that the estimates in the region $I V$ in $\mathrm{Fi}$ gure 3 determined by $L^{2} \times L^{2} \rightarrow L^{2}, L^{4} \times L^{4} \rightarrow L^{4}$ and $L^{1} \times L^{1} \rightarrow L^{\infty}$ are sharp up to $R^{\varepsilon}$ by using the idea of Knapp example. We will only do computations for the estimate when $p=q=2$.

Example $4.7(\mathbf{I V})$. If $R \geq 1 / M, R^{-\frac{n-2}{2}}$ is best possible in the following inequality.

$$
\left\|\left(f d \sigma_{1}\right)^{\vee}\left(g d \sigma_{2}\right)^{\vee}\right\|_{L_{t, x}^{2}} \lesssim R^{-\frac{n-2}{2}}\|f\|_{L^{2}\left(S_{1}\right)}\|g\|_{L^{2}\left(S_{2}\right)} .
$$

We take

$$
\begin{aligned}
& f\left(|\xi|^{2}, \xi\right)=|\xi|^{-\frac{n-2}{2}} e^{i\left(-r_{0}|\xi|+t_{0}|\xi|^{2}\right)} 1_{\left\{1 \leq|\xi| \leq 1+M^{1 / 2}\right\}}, \\
& g\left(|\eta|^{2}, \eta\right)=|\eta|^{-\frac{n-2}{2}} e^{i\left(-r_{0}|\eta|+t_{0}|\eta|^{2}\right)} 1_{\{M \leq|\eta| \leq 2 M\}},
\end{aligned}
$$

where $r_{0} \in[R / 2, R]$ and $t_{0} \in \mathbb{R}$. By the heuristic approximation (3.6), the left-hand side of (4.18) is comparable to

$$
R^{-\frac{n-2}{2}}\left(\int_{R / 2}^{R} \int_{\mathbb{R}}\left|\int_{1}^{1+M^{\frac{1}{2}}} e^{i\left[\left( \pm r-r_{0}\right) s_{1}-\left(t-t_{0}\right) s_{1}^{2}\right]} d s_{1} \int_{I_{M}} e^{i\left[\left( \pm r-r_{0}\right) s_{2}-\left(t-t_{0}\right) s_{2}^{2}\right]} d s_{2}\right|^{2} d t d r\right)^{1 / 2}
$$

We choose $r \in[R / 2, R]$ and $t \in \mathbb{R}$ such that

$$
\begin{aligned}
\left|\left(r-r_{0}\right)-2\left(t-t_{0}\right)\right| & \leq M^{-1 / 2}, \\
\left|\left(r-r_{0}\right)-2 M\left(t-t_{0}\right)\right| & \leq M^{-1}, \\
M^{-1} / 100 \leq t-t_{0} & \leq M^{-1} / 50,
\end{aligned}
$$


i.e., $r$ and $t$ are located in the intersection area of two tubes which has size $M^{-1} \times M^{-1 / 2}$. Then by similar discussions as Example 4.4, the left-hand side of $(4.18) \gtrsim R^{-(n-2) / 2} M^{3 / 4}$; by direct computations the right-hand side of $(4.18) \lesssim R^{-(n-2) / 2} M^{3 / 4}$. Hence we see that the estimate $L^{2} \times L^{2} \rightarrow L^{2}$ when $R \geq 1 / M$ is sharp.

The next example shows that the estimates in the region $V$ in Figure 3 determined by the lines $q=4, q=\infty$ and $q=3 p^{\prime}$ are sharp. In this example, we will do the computations for the estimates on the line $q=\infty$.

Example $4.8(\mathbf{V})$. If $R \geq 1 / M, R^{-(n-2)} \times M^{\frac{n}{2}-\frac{n-1}{p}}$ is best possible in the following inequality

$$
\left\|\left(f d \sigma_{1}\right)^{\vee}\left(g d \sigma_{2}\right)^{\vee}\right\|_{L_{t, x}^{\infty}} \lesssim R^{-(n-2)} M^{\frac{n}{2}-\frac{n-1}{p}}\|f\|_{L^{p}\left(S_{1}\right)}\|g\|_{L^{p}\left(S_{2}\right)},
$$

where $1 \leq p \leq \infty$.

We take

$$
\begin{aligned}
& f\left(|\xi|^{2}, \xi\right)=|\xi|^{-\frac{n-2}{2}} e^{i\left(-r_{0}|\xi|+t_{0}|\xi|^{2}\right)} 1_{\{1 \leq|\xi| \leq 2\}}, \\
& g\left(|\eta|^{2}, \eta\right)=|\eta|^{-\frac{n-2}{2}} e^{i\left(-r_{0}|\eta|+t_{0}|\eta|^{2}\right)} 1_{\{M \leq|\eta| \leq 2 M\}},
\end{aligned}
$$

where $r_{0} \in[R / 2, R]$ and $t_{0} \in \mathbb{R}$ satisfying the $L^{\infty}$ norms $\left\|(f d \sigma)^{\vee}\right\|_{L_{t, x}^{\infty}\left(\mathbb{R} \times A_{R}\right)}$ and $\left\|(g d \sigma)^{\vee}\right\|_{L_{t, x}^{\infty}\left(\mathbb{R} \times A_{R}\right)}$ can be realized at $\left(t_{0}, x_{0}\right)$ with $\left|x_{0}\right|=r_{0}$. By the heuristic approximation (3.6), the left-hand side of (4.19) is comparable to

$$
R^{-(n-2)}\left|\int_{I_{1}} e^{i\left( \pm r_{0}-r_{0}\right) s_{1}} d s_{1} \int_{I_{M}} e^{i\left( \pm r_{0}-r_{0}\right) s_{2}} d s_{2}\right| .
$$

Then by the same reasoning as Example 3.10, the above $\gtrsim R^{-(n-2)} M$. On the other hand, the right-hand side of $(4.19) \lesssim R^{-(n-2)} M$. Hence the estimates on the line $q=\infty$ when $R \geq 1 / M$ are sharp.

For lines $q=4,4 \leq p \leq \infty$ or $q=3 p^{\prime}, 1 \leq p<4$, the estimates go back to (4.9). We will choose $r \in[R / 2, R]$ and $t$ such that $2 \leq r-r_{0} \leq 4$ and $2 \leq t-t_{0} \leq 4$. Then by similar reasoning, the estimates on these lines are sharp.

\section{Case 2: $2 \leq R \leq 1 / M$.}

In this subcase, we will construct counterexamples to show the restriction estimates in Theorem 2.5 are sharp when $2 \leq R \leq 1 / M$. As in the Case 1, we will start with a "narrow" example which shows that estimates in the region $I$ in Figure 3 determined by $L^{2} \times L^{2} \rightarrow L^{1}, L^{2} \times L^{2} \rightarrow L^{2}$ and $L^{1} \times L^{1} \rightarrow L^{\infty}$ are sharp. In this example, we will do computations for the estimate $L^{2} \times L^{2} \rightarrow L^{1}$. 
Example 4.9 (I). If $2 \leq R \leq 1 / M, R^{\frac{n}{2}} M^{\frac{n-3}{2}}$ is best possible in

$$
\left\|\left(f d \sigma_{1}\right)^{\vee}\left(g d \sigma_{2}\right)^{\vee}\right\|_{L_{t, x}^{1}} \lesssim R^{\frac{n}{2}} M^{\frac{n-3}{2}}\|f\|_{L^{2}\left(S_{1}\right)}\|g\|_{L^{2}\left(S_{2}\right)} .
$$

We take

$$
\begin{aligned}
& f\left(|\xi|^{2}, \xi\right)=|\xi|^{-\frac{n-2}{2}} e^{i\left(-r_{0}|\xi|+t_{0}|\xi|^{2}\right)} 1_{\left\{1 \leq|\xi| \leq 1+M^{2}\right\}}, \\
& g\left(|\eta|^{2}, \eta\right)=|\eta|^{-(n-2)} e^{i t_{0}|\eta|^{2}} 1_{I_{M}},
\end{aligned}
$$

where $r_{0} \in[R / 2, R]$ and $t_{0} \in \mathbb{R}$. By the heuristic approximation (3.6) only for $(f d \sigma)^{\vee}$, we see that the left-hand side of (4.20) is comparable to

$$
R^{\frac{n-2}{2}} \int_{R / 2}^{R} \int_{\mathbb{R}}\left|\int_{1}^{1+M^{2}} e^{i\left(\left( \pm r-r_{0}\right) s_{1}-\left(t-t_{0}\right) s_{1}^{2}\right)} d s_{1} \int_{I_{M}} e^{-i\left(t-t_{0}\right) s_{2}^{2}}(d \mu)^{\vee}\left(r s e_{1}\right) d s_{2}\right| d t d r .
$$

We choose $r \in[R / 2, R]$ and $t \in \mathbb{R}$ such that

$$
R / 100 \leq r-r_{0} \leq R / 50, M^{-2} / 100 \leq t-t_{0} \leq M^{-2} / 50 .
$$

Then we have $\left|\left[\left(r-r_{0}\right)-2\left(t-t_{0}\right)\right]\left(s_{1}-1\right)\right| \leq c$ with a small $c>0$ and $-\frac{\left(r+r_{0}\right)+2\left(t-t_{0}\right)}{2\left(t-t_{0}\right)}<-1$. From the principle of non-stationary phase and the triangle inequality, the left-hand side of $(4.20) \gtrsim R^{\frac{n}{2}} M$. On the other hand, the right-hand side of $(4.20) \lesssim R^{\frac{n}{2}} M$. Thus the estimate $L^{2} \times L^{2} \rightarrow L^{1}$ when $2 \leq R \leq 1 / M$ is sharp.

In the next example, we are going to show the estimates in the region $I I$ in Figure 3 determined by the lines $q=1$ and $q=2$ are sharp. In this example, we will do computations for the estimates on the line $q=1$.

Example 4.10 (II). If $2 \leq R \leq 1 / M, R^{\frac{n}{2}} M^{-1+\frac{n-1}{p^{\prime}}}$ is best possible in the following inequality

$$
\left\|\left(f d \sigma_{1}\right)^{\vee}\left(g d \sigma_{2}\right)^{\vee}\right\|_{L_{t, x}^{1}} \lesssim R^{\frac{n}{2}} M^{-1+\frac{n-1}{p^{\prime}}}\|f\|_{L^{p}\left(S_{1}\right)}\|g\|_{L^{p}\left(S_{2}\right)}
$$

where $2 \leq p \leq \infty$. We define an index set $J:=\left\{j: 1 \leq j \leq\left[M^{-2}\right]\right\}$. For each $j \in J$, we set

$$
f_{j}\left(|\xi|^{2}, \xi\right)=F_{j}(|\xi|)=|\xi|^{-\frac{n-2}{2}} e^{i\left(-r_{0}|\xi|+t_{0}|\xi|^{2}\right)} 1_{\left\{1+(j-1) M^{2} \leq|\xi| \leq 1+j M^{2}\right\}} .
$$

Then we define

$$
f=\sum_{j} \varepsilon_{j} f_{j}, g\left(|\eta|^{2}, \eta\right)=|\eta|^{-(n-2)} e^{i t_{0}|\eta|^{2}} 1_{I_{M}},
$$

where $\left\{\varepsilon_{j}: j \in J\right\}$ is a set of i.i.d. random variables taking \pm 1 with an equal probability $1 / 2$. 
By using the Khintchine inequality, we obtain

$$
\mathbb{E}\left(\left\|\left(f d \sigma_{1}\right)^{\vee}\left(g d \sigma_{2}\right)^{\vee}\right\|_{L_{t, x}^{1}\left(\mathbb{R} \times A_{R}\right)}\right) \sim\left\|\left(\sum_{j}\left|\left(f_{j} d \sigma_{1}\right)^{\vee}\left(g d \sigma_{2}\right)^{\vee}\right|^{2}\right)^{1 / 2}\right\|_{L_{t, x}^{1}\left(\mathbb{R} \times A_{R}\right)},
$$

where $\mathbb{E}(X)$ denotes the expectation of the random variable $X$. By the heuristic approximation (3.6), the right-hand side of the above is comparable to

$$
\begin{aligned}
R^{\frac{n-2}{2}} \int_{R / 2}^{R} \int_{\mathbb{R}}\left(\sum_{j} \mid \int_{c_{j}-M^{2}}^{c_{j}} e^{i\left[\left( \pm r-r_{0}\right) s_{1}-\left(t-t_{0}\right) s_{1}^{2}\right]} d s_{1} \times\right. \\
\left.\quad \times\left.\int_{I_{M}} e^{-i\left(t-t_{0}\right) s_{2}^{2}}(d \mu)^{\vee}\left(r s_{2} e_{1}\right) d s_{2}\right|^{2}\right)^{1 / 2} d t d r
\end{aligned}
$$

where $c_{j}=1+j M^{2}$. We choose $r \in[R / 2, R]$ and $t \in \mathbb{R}$ such that

$$
R / 100 \leq r-r_{0} \leq R / 50, M^{-2} / 100 \leq t-t_{0} \leq M^{-2} / 50 .
$$

This gives $\left|\left[\left(r-r_{0}\right) 2\left(t-t_{0}\right)\right]\left(s_{1}-c_{j}\right)\right| \leq c$ and $-\frac{\left(r+r_{0}\right)+2\left(t-t_{0}\right)}{2\left(t-t_{0}\right)}<-1$. Then by the principle of non-stationary phase and the triangle inequality, the above is bounded below by $R M^{-2}|J|^{1 / 2} M^{3}$, where $|J| \sim M^{-2}$ denotes the cardinality of the set $J$. Hence the left-hand side of $(4.21) \gtrsim R^{n / 2}$ and the right-hand side of $(4.21) \lesssim R^{n / 2}$ for $2 \leq p \leq \infty$. Thus the estimates on the line $q=1$ when $2 \leq R \leq 1 / M$ are sharp.

In the following example, we will see the estimates in the region III in Figure 3 determined by the lines $q=2$ and $q=4$ are sharp in the case $2 \leq R \leq 1 / M$. We will do computations for the estimates on the line $q=2$ below.

Example 4.11 (III). If $2 \leq R \leq 1 / M, R^{1 / 2} M^{(n-1) / p^{\prime}}$ is best possible in the following inequality.

$$
\left\|\left(f d \sigma_{1}\right)^{\vee}\left(g d \sigma_{2}\right)^{\vee}\right\|_{L_{t, x}^{2}} \lesssim R^{1 / 2} M^{(n-1) / p^{\prime}}\|f\|_{L^{p}\left(S_{1}\right)}\|g\|_{L^{p}\left(S_{2}\right)},
$$

where $2 \leq p \leq \infty$.

We take

$$
\begin{aligned}
& f\left(|\xi|^{2}, \xi\right)=|\xi|^{-\frac{n-2}{2}} e^{i\left(-r_{0}|\xi|+t_{0}|\xi|^{2}\right)} 1_{\{1 \leq|\xi| \leq 2\}}, \\
& g\left(|\eta|^{2}, \eta\right)=|\eta|^{-(n-2)} e^{i t_{0}|\eta|^{2}} 1_{I_{M}},
\end{aligned}
$$

where $r_{0} \in[R / 2, R]$ and $t_{0} \in \mathbb{R}$. Then by the heuristic approximation (3.6), the left-hand side of (4.22) is comparable to

$$
\left(\int_{R / 2}^{R} \int_{\mathbb{R}}\left|\int_{I_{1}} e^{i\left[\left( \pm r-r_{0}\right) s_{1}-\left(t-t_{0}\right) s_{1}^{2}\right]} d s_{1} \int_{I_{M}} e^{-i\left(t-t_{0}\right) s_{2}^{2}}(d \mu)^{\vee}\left(r s_{2} e_{1}\right) d s_{2}\right|^{2} d t d r\right)^{1 / 2} \text {. }
$$


We choose $r \in[R / 100, R / 50]$ and $t \in \mathbb{R}$ such that $\frac{r-r_{0}}{2\left(t-t_{0}\right)} \in I_{1}$. Then $r$ and $t$ are in the region of size $\sim R^{2}$. The principles of stationary phase and non-stationary phase again give, for any $N \geq 0$,

$$
\left|\int_{I_{1}} e^{i\left[\left(r-r_{0}\right) s_{1}-\left(t-t_{0}\right) s_{1}^{2}\right]} d s_{1}\right| \gtrsim R^{-\frac{1}{2}},\left|\int_{I_{1}} e^{-i\left[\left(r+r_{0}\right) s_{1}+\left(t-t_{0}\right) s_{1}^{2}\right]} d s_{1}\right| \lesssim_{N} R^{-N} .
$$

With this choice of $r$ and $t$, we have $\left|\int_{I_{M}} e^{-i\left(t-t_{0}\right) s_{2}^{2}}(d \mu)^{\vee}\left(i r s_{2} \omega\right) d s_{2}\right| \gtrsim M$. Hence from the triangle inequality, the left-hand side of $(4.22) \gtrsim R^{1 / 2} M$. On the other hand, the right-hand side of $(4.22) \lesssim R^{1 / 2} M$. Thus we see that the estimates on the line $q=2$ when $2 \leq R \leq 1 / M$ are sharp.

The next example will show that the estimates in the region $I V$ in $\mathrm{Fi}$ gure 3 determined by $L^{2} \times L^{2} \rightarrow L^{2}, L^{4} \times L^{4} \rightarrow L^{4}$ and $L^{1} \times L^{1} \rightarrow L^{\infty}$ are sharp. We will do the computations for the estimate $L^{2} \times L^{2} \rightarrow L^{2}$.

Example $4.12(\mathbf{I V})$. If $2 \leq R \leq 1 / M, R^{1 / 2} M^{(n-1) / 2}$ is best possible in the following inequality.

$$
\left\|\left(f d \sigma_{1}\right)^{\vee}\left(g d \sigma_{2}\right)^{\vee}\right\|_{L_{t, x}^{2}} \lesssim R^{\frac{1}{2}} M^{\frac{n-1}{2}}\|f\|_{L^{2}\left(S_{1}\right)}\|g\|_{L^{2}\left(S_{2}\right)} .
$$

We take

$$
\begin{aligned}
& f\left(|\xi|^{2}, \xi\right)=|\xi|^{-\frac{n-2}{2}} e^{i\left(-r_{0}|\xi|+t_{0}|\xi|^{2}\right)} 1_{\left\{1 \leq|\xi| \leq 1+R^{-1 / 2}\right\}}, \\
& g\left(|\eta|^{2}, \eta\right)=|\eta|^{-(n-2)} e^{i t_{0}|\eta|^{2}} 1_{I_{M}},
\end{aligned}
$$

where $r_{0} \in[R / 2, R]$ and $t_{0} \in \mathbb{R}$. By the heuristic approximation for $(f d \sigma)^{\vee}$, the left-hand side of (4.23) is comparable to

$$
\left(\int_{R / 2}^{R} \int_{\mathbb{R}}\left|\int_{1}^{1+R^{-1 / 2}} e^{i\left[\left( \pm r-r_{0}\right) s_{1}-\left(t-t_{0}\right) s_{1}^{2}\right]} d s_{1} \int_{I_{M}} e^{-i\left(t-t_{0}\right) s_{2}^{2}}(d \mu)^{\vee}\left(i r s_{2} e_{1}\right) d s_{2}\right|^{2} d t d r\right)^{1 / 2} .
$$

We choose $r \in[R / 2, R]$ and $t \in \mathbb{R}$ such that

$$
\left|\left(r-r_{0}\right)-2\left(t-t_{0}\right)\right| \leq R^{1 / 2} / 100, R^{1 / 2} / 100 \leq t-t_{0} \leq R^{1 / 2} / 50 .
$$

Then $r$ and $t$ are located in the intersection area of two tubes which has size of $R \times R^{1 / 2}$. Hence the left-hand side of $(4.23) \gtrsim R^{1 / 4} M$. On the other hand, its right-hand side $\lesssim R^{1 / 4} M$. Thus we see that the estimate $L^{2} \times L^{2} \rightarrow L^{2}$ when $2 \leq R \leq 1 / M$ is sharp.

The following example will show that the estimates in the region $V$ in Figure 3 determined by the lines $q=4, q=\infty$ and $q=3 p^{\prime}$ are sharp. 
Example $4.13(\mathbf{V})$. If $2 \leq R \leq 1 / M, R^{-(n-2) / 2} M^{(n-1) / p^{\prime}}$ is best possible in the following inequality

$$
\left\|\left(f d \sigma_{1}\right)^{\vee}\left(g d \sigma_{2}\right)^{\vee}\right\|_{L_{t, x}^{\infty}} \lesssim R^{-\frac{n-2}{2}} M^{\frac{n-1}{p^{\prime}}}\|f\|_{L^{p}\left(S_{1}\right)}\|g\|_{L^{p}\left(S_{2}\right)},
$$

where $1 \leq p \leq \infty$.

We take

$$
\begin{aligned}
& f\left(|\xi|^{2}, \xi\right)=|\xi|^{-\frac{n-2}{2}} e^{i\left(-r_{0}|\xi|+t_{0}|\xi|^{2}\right)} 1_{\{1 \leq|\xi| \leq 2\}}, \\
& g\left(|\eta|^{2}, \eta\right)=|\eta|^{-(n-2)} e^{i t_{0}|\eta|^{2}} 1_{I_{M}},
\end{aligned}
$$

where $r_{0} \in[R / 2, R]$ and $t_{0} \in \mathbb{R}$. They are chosen such that $\left\|\left(f d \sigma_{1}\right)^{\vee}\right\|_{L_{t x}^{\infty}}$ and $\left\|\left(g d \sigma_{2}\right)^{\vee}\right\|_{L_{t, x}^{\infty}}$ can be realized at $\left(t_{0}, x_{0}\right)$ with $\left|x_{0}\right|=r_{0}$. By the heuristic approximation (3.6),

$$
R^{-\frac{n-2}{2}}\left|\int_{I_{1}} e^{i\left( \pm r_{0}-r_{0}\right) s_{1}} d s_{1} \int_{I_{M}}(d \mu)^{\vee}\left(r s_{2} e_{1}\right) d s_{2}\right|
$$

Then from the triangle inequality, the left-hand side of $(4.24) \gtrsim R^{-(n-2) / 2} M$. On the other hand, its right-hand side $\lesssim R^{-(n-2) / 2} M$ for $1 \leq p \leq \infty$. Thus the estimates on the line $q=\infty$ when $2 \leq R \leq 1 / M$ are sharp.

When $q=4,4 \leq p \leq \infty$, or $q=3 p^{\prime}, 1 \leq p<4$, the estimates go back to (4.6). We choose $r \in[R / 2, R]$ and $t \in \mathbb{R}$ such that $2 \leq r-r_{0} \leq 4$ and $2 \leq t-t_{0} \leq 4$.

\section{Case 3: $R \leq 1$.}

In this subcase, we will construct counterexamples to show the estimates (4.1) and (4.2) are sharp. We will omit the computations for simplicity.

The following example shows that the estimates in the region $I$ determined by $L^{2} \times L^{2} \rightarrow L^{1}, L^{2} \times L^{2} \rightarrow L^{2}$ and $L^{1} \times L^{1} \rightarrow L^{\infty}$ are sharp.

Example $4.14(\mathrm{I})$. We take

$$
\begin{aligned}
& f\left(|\xi|^{2}, \xi\right)=F(|\xi|)=|\xi|^{-(n-2)} e^{i t_{0}|\xi|^{2}} 1_{\left\{1 \leq|\xi| \leq 1+M^{2}\right\}} \\
& g\left(|\eta|^{2}, \eta\right)=G(|\eta|)=|\eta|^{-(n-2)} e^{i t_{0}|\eta|^{2}} 1_{I_{M}}
\end{aligned}
$$

where $t_{0} \in \mathbb{R}$. The $r$ and $t$ are chosen such that $\frac{R}{2} \leq r \leq R$ and $\frac{1}{100 M^{2}} \leq$ $t-t_{0} \leq \frac{1}{50 M^{2}}$.

The next example shows that the estimates in the region $I I$ determined by the lines $q=1$ and $q=2$ are sharp. 
Example 4.15 (II). We define an index set $J:=\left\{j: 1 \leq j \leq\left[M^{-2}\right]\right\}$. For each $j \in J$, we set

$$
f_{j}\left(|\xi|^{2}, \xi\right)=F_{j}(|\xi|)=|\xi|^{-(n-2)} e^{i t_{0}|\xi|^{2}} 1_{\left\{1+(j-1) M^{2} \leq|\xi| \leq 1+j M^{2}\right\}} .
$$

Then we define

$$
f=\sum_{j} \varepsilon_{j} f_{j}, g\left(|\eta|^{2}, \eta\right)=|\eta|^{-(n-2)} e^{i t_{0}|\eta|^{2}} 1_{I_{M}}
$$

where $\left\{\varepsilon_{j}: j \in J\right\}$ is a set of i.i.d. random variables taking \pm 1 with an equal probability $1 / 2$, and the $r$ and $t$ are chosen such that $R / 2 \leq r \leq R$ and $1 / 2 \leq t-t_{0} \leq 1$.

The third example shows that the estimate (4.2) is sharp. Hence the estimates in the regions $I I I, I V$ and $V$ when $R \leq 1$ are sharp.

Example 4.16 (III, IV, V). We take

$$
\begin{aligned}
& f\left(|\xi|^{2}, \xi\right)=F(|\xi|)=|\xi|^{-(n-2)} e^{i t_{0}|\xi|^{2}} 1_{I_{1}}, \\
& g\left(|\eta|^{2}, \eta\right)=G(|\eta|)=|\eta|^{-(n-2)} e^{i t_{0}|\eta|^{2}} 1_{I_{M}},
\end{aligned}
$$

where $t_{0} \in \mathbb{R}$. The $r$ and $t$ will be are chosen such that $\frac{R}{2} \leq r \leq R$ and $\frac{1}{2} \leq t-t_{0} \leq 1$.

Thus the proof of Theorem 2.5 is complete.

\section{Connection with the restriction estimates for the sphere or the hypersurface of elliptic type}

In this section we are concerned with whether the analogous results of Theorems 2.1 and 2.5 remain valid if $S$ is replaced with the lower third of the sphere $S^{n-1}$ or a cylindrically symmetric and compact hypersurface of elliptic type.

Let us first consider the case where the paraboloid is replaced by the sphere $\mathcal{S}^{n-1}$ in $\mathbb{R}^{n}$. Suppose $f$ is a cylindrically symmetric function supported on a compact set of $\mathcal{S}^{n-1}, S:=\left\{\left(-\sqrt{1-|\xi|^{2}}, \xi\right) \in \mathbb{R} \times \mathbb{R}^{n-1}: M \leq\right.$ $|\xi| \leq 2 M\}$, where $0<M \leq 1 / 6$. Then

$$
(f d \mu)^{\vee}(t, x)=\int_{M \leq|\xi| \leq 2 M} e^{i\left(x \cdot \xi-t \sqrt{1-|\xi|^{2}}\right)} F(|\xi|) d \xi,
$$

where $d \mu$ is the surface measure of the sphere and $F(|\xi|)=f\left(-\sqrt{1-|\xi|^{2}}, \xi\right)$. 
Since $f$ is cylindrically symmetric, we see that $(f d \mu)^{\vee}$ is also cylindrically symmetric. Then if we change (5.1) to the polar coordinates to obtain

$$
(f d \mu)^{\vee}(t, r)=\int_{I} F(s) e^{-i t \sqrt{1-s^{2}}}(d \mu)^{\vee}\left(r s e_{1}\right) s^{n-2} d s,
$$

where $I=[M, 2 M]$. By the Taylor expansion of $\sqrt{1-s^{2}}$ at $s=0$,

$$
-\sqrt{1-s^{2}}=-1+\frac{1}{2} s^{2}+C_{1}(s) s^{4},
$$

where $C_{1}(s) \sim 1$ for all $s \in I$. Then from (5.2),

$$
\left|(f d \mu)^{\vee}(2 t, r)\right|=\left|\int_{I} F(s) e^{i t\left(s^{2}+C_{2} s^{4}\right)}(d \mu)^{\vee}\left(r s e_{1}\right) s^{n-2} d s\right|,
$$

where $C_{2}(s) \sim 1$ for all $s \in I$. The factor " 2 " in $(f d \mu)^{\vee}(2 t, r)$ is artificial since we are going to integrate $t$ in $\mathbb{R}$. We make two key observations similar to those we used in Theorem 2.1 and 2.5 as follows.

- Since $0 \leq M \leq 1 / 6$,

$$
\frac{d\left(s^{2}+C_{2}(s) s^{4}\right)}{d s} \sim s, \frac{d^{2}\left(s^{2}+C_{2}(s) s^{4}\right)}{d^{2} s} \sim 1 .
$$

Heuristically, this condition means that if we change variables $s^{2}+$ $C_{2}(s) s^{4} \rightarrow a$, it is almost like changing $s^{2} \rightarrow a$. Hence the analogous result to Proposition 3.6 will hold for the lower third of the sphere.

- Form the geometric properties of the sphere,

$$
\left\|d \mu_{1} * d \mu_{2}\right\|_{L_{t, r}^{\infty}} \lesssim O(1),
$$

when $d \mu_{1}$ and $d \mu_{2}$ are the canonical Lebesgue measure of two arcs of size $O(1)$ supported on the sphere $\mathcal{S}^{1}$ but separated by a distance $O(1)$. Hence the analogous result to Proposition 3.5 will hold.

Those observations enable us to run all the arguments in Theorems 2.1 and 2.5.

We now turn to the case where $S$ is replaced by a cylindrically symmetric and compact hypersurface $S$ of elliptic type, i.e., $S$ is of the form

$$
S:=\left\{(\tau, \xi) \in \mathbb{R} \times \mathbb{R}^{n-1}: \tau=|\xi|^{2}+\varepsilon \phi(\xi)\right\}
$$

where the error function $\phi(\xi)$ is radial and smooth, and $\varepsilon$ is a sufficiently small parameter depending on the smooth norms of $\phi$ and on the size of $S$, or more generally on the separation of $S_{1}$ and $S_{2}$. In other words, $S$ is the small perturbation of the standard paraboloid. By similar observations we made on the sphere, we can establish the analogous results to Theorems 2.1 and 2.5 for the cylindrically symmetric functions compactly supported on $S$ defined in (5.5). 


\section{Connection with Strichartz inequalities of the Schrö- dinger equation}

\subsection{Linear Strichartz estimates}

The restriction problem is closely related to that of estimating solutions to linear PDE such as the wave equation and the Schrödinger equation. Strichartz first observed this connection in [16], which initiated the intensive study on various Strichartz estimates. In this section we will interpret our restriction estimates regarding $(f d \sigma)^{\vee}$ in terms of the solutions to the Schrödinger equations.

Suppose $f(\tau, \xi)$ is a function supported on the paraboloid $S$ in $\mathbb{R} \times \mathbb{R}^{n-1}$. Functions of the form $u(t, x):=(f d \sigma)^{\vee}$, where $d \sigma$ is the canonical Lebesgue measure on $S$, can be easily seen to solve the free Schrödinger equation

$$
i u_{t}+\triangle u=0, u(0, x)=u_{0}(x),
$$

where the spatial Fourier transform $\hat{u}_{0}(\xi)=f\left(|\xi|^{2}, \xi\right)$. It is easy to deduce that $f$ is cylindrically symmetric on $\mathbb{R} \times \mathbb{R}^{n-1}$ if $u_{0}$ is radial on $\mathbb{R}^{n-1}$. By this interpretation, the linear estimate $L^{p} \rightarrow L^{q}$ or the bilinear restriction estimate $L^{p} \times L^{p} \rightarrow L^{q}$ will correspond to certain Strichartz estimates. For instance, the Tomas-Stein restriction estimate $L^{2} \rightarrow L^{2(n+1) /(n-1)}$ implies the Strichartz estimate

$$
\left\|e^{i t \triangle} u_{0}\right\|_{L_{t, x}^{\frac{2(n+1)}{n-1}}\left(\mathbb{R} \times \mathbb{R}^{n-1}\right)} \lesssim\left\|u_{0}\right\|_{L^{2}\left(\mathbb{R}^{n-1}\right)},
$$

where we have denoted $u$ by $e^{i t \triangle} u_{0}(x)=\int_{\mathbb{R}^{n-1}} e^{i\left(x \xi+t|\xi|^{2}\right)} \hat{u}_{0}(\xi) d \xi$. This is known to be best possible simply by the scaling property associated to the Schrödinger equation. In fact, we have the following optimal result called the linear Strichartz estimates [8],

$$
\left\|e^{i t \triangle} u_{0}\right\|_{L_{t}^{q} L_{x}^{r}\left(\mathbb{R} \times \mathbb{R}^{n-1}\right)} \lesssim\left\|u_{0}\right\|_{L^{2}\left(\mathbb{R}^{n-1}\right)}
$$

if and only if

$$
\frac{2}{q}+\frac{n-1}{r}=\frac{n-1}{2}, \quad q \geq 2, r \geq 2,(q, r, n) \neq(2, \infty, 3) .
$$

A natural question arises: if we assume that $\hat{u}_{0}$ is radial and supported on a compact set $U:=\left\{\xi \in \mathbb{R}^{n-1}: M \leq|\xi| \leq 2 M\right\}$ with dyadic $M>0$, do we have further estimates available? The answer is confirmed in Corollary 2.3. In particular, we have

Corollary 6.2. Suppose $u_{0}$ is defined as above. Then for any $q>\frac{4 n-2}{2 n-3}$,

$$
\left\|e^{i t \triangle} u_{0}\right\|_{L_{t, x}^{q}\left(\mathbb{R} \times \mathbb{R}^{n-1}\right)} \lesssim M^{\frac{n-1}{2}-\frac{n+1}{q}}\left\|u_{0}\right\|_{L^{2}\left(\mathbb{R}^{n-1}\right)} .
$$


Remark 6.3. For such functions, one can easily extend the current range (6.3) for the linear mixed norm Strichartz estimates (6.2) by interpolating them with the estimate (6.4).

In another direction, one can also obtain various weighted Strichartz estimates. This type of estimates for radial data has proven very useful in establishing the global well-posedness and scattering results for certain Schrödinger equations, see e.g., [24]. In [26], Vilela showed that, assuming $u_{0} \in L^{2}\left(\mathbb{R}^{n-1}\right)$ to be radial,

$$
\left\|D_{x}^{s} e^{i t \triangle} u_{0}\right\|_{L_{t, x}^{2}\left(|x|^{-\alpha}\right)} \lesssim\left\|u_{0}\right\|_{L^{2}}
$$

if and only if $\alpha=2(1-s), 1<\alpha<n-1$ and $n \geq 3$, where $D_{x}^{s} f$ is defined via the spatial Fourier transform by $\widehat{D_{x}^{s} f}(\xi)=|\xi|^{s} \hat{f}(\xi)$. The "only if" part is given in [26] by the decay estimate of $(d \sigma)^{\vee}$ and scaling. Here we will give another proof of the "if" part by using the linear dyadic restriction restriction estimates given by Theorem 2.1.

Proof. We first assume that $u_{0}$ has dyadically localized frequency, i.e., $\hat{u}_{0}$ supported on the set $\{\xi: M / 2 \leq|\xi| \leq M\}$ with dyadic $M$. Then we set $f\left(|\xi|^{2}, \xi\right)=\hat{u}_{0}(M \xi)$, i.e., $f \in \mathcal{L}_{1}$. Then from the estimate $L^{2} \rightarrow L^{2}$ in Theorem 2.1, we obtain, $\forall \varepsilon>0$,

$$
\left\||x|^{-(1+\varepsilon) / 2}(f d \sigma)^{\vee}\right\|_{L^{2}(\mathbb{R} \times\{|x| \geq 1\})} \lesssim_{\varepsilon}\|f\|_{L^{2}\left(\mathbb{R}^{n-1}\right)} .
$$

If we restrict $0<\varepsilon<n-2$, by the Plancherel theorem in $t$,

$$
\left\||x|^{-(1+\varepsilon) / 2}(f d \sigma)^{\vee}\right\|_{L^{2}(\mathbb{R} \times\{|x| \leq 1\})} \lesssim \varepsilon\|f\|_{L^{2}\left(\mathbb{R}^{n-1}\right)} .
$$

Hence

$$
\left\||x|^{-(1+\varepsilon) / 2}(f d \sigma)^{\vee}\right\|_{L^{2}\left(\mathbb{R} \times \mathbb{R}^{n-1}\right)} \lesssim \varepsilon\|f\|_{L^{2}\left(\mathbb{R}^{n-1}\right)} .
$$

By re-scaling by $M$,

$$
\left\||x|^{-(1+\varepsilon) / 2} M^{(1-\varepsilon) / 2} e^{i t \triangle} u_{0}\right\|_{L^{2}\left(\mathbb{R} \times \mathbb{R}^{n-1}\right)} \lesssim \varepsilon\left\|u_{0}\right\|_{L^{2}\left(\mathbb{R}^{n-1}\right)}
$$

By the weighted Hörmander-Mikhlin theorem [24, Lemma 2.2],

$$
\left\|D^{(1-\varepsilon) / 2} e^{i t \triangle} u_{0}\right\|_{L^{2}\left(|x|^{-(1+\varepsilon)}\right)} \lesssim_{\varepsilon}\left\|u_{0}\right\|_{L^{2}\left(\mathbb{R}^{n-1}\right)} .
$$

Setting $s=(1-\varepsilon) / 2$ and $\alpha=1+\varepsilon$, we obtain (6.5) for frequency localized $u_{0}$. Then we follow the approach of using the Khintchine inequality to prove the Littlewood-Paley inequality and use the weighted inequalities for singular integrals[14, Chapter 5, Corrollary 4.2] $\left(|x|^{-\alpha}\right.$ is a $A_{2}$ weight) to obtain (6.5). 


\subsection{Bilinear Strichartz estimates}

Form the linear Strichartz estimates (6.2), we see their bilinear analogues,

$$
\left\|e^{i t \triangle} u_{0} e^{i t \triangle} v_{0}\right\|_{L_{t}^{q} L_{x}^{r}\left(\mathbb{R} \times \mathbb{R}^{n-1}\right)} \lesssim\left\|u_{0}\right\|_{L^{2}\left(\mathbb{R}^{n-1}\right)}\left\|v_{0}\right\|_{L^{2}\left(\mathbb{R}^{n-1}\right)}
$$

if and only if

$$
\frac{2}{q}+\frac{n-1}{r}=n-1 ; \quad q, r \geq 1 ;(q, r, n) \neq(1, \infty, 3) .
$$

For the necessity of excluding the endpoint $(1, \infty, 3)$, see [21].

The estimate (6.6) becomes more interesting when we assume $u_{0}$ and $v_{0}$ are compactly supported and separated by a distance comparable to $O(1)$. In this case, we expect that there are more estimates available. For instance, when $q=r$, Klainerman and Machedon [9] conjectured that (6.6) holds if and only if $q=r \geq(n+2) / n$. The exponent $(n+2) / n$ is best possible, see e.g., [23], [20]. This conjecture has been verified by Tao in [20] up to the endpoint $(n+2) / n$. The analogous results in the cone setting were established by Wolff in the non-endpoint case [28] and Tao in the endpoint case [19].

As shown in Corollary 2.7, we have further estimates available if we assume that $\widehat{u}_{0}$ and $\widehat{v}_{0}$ are radial functions and compactly supported on $U_{1}=$ $\left\{\xi \in \mathbb{R}^{n-1}: M_{1} / 2 \leq|\xi| \leq M_{1}\right\}$ and $U_{2}=\left\{\left(\xi \in \mathbb{R}^{n-1}: M_{2} / 2 \leq|\xi| \leq M_{2}\right\}\right.$, respectively. Here $M_{1}>0, M_{2}>0$ are dyadic numbers satisfying $M_{2} \leq$ $M_{1} / 4$. For instance, as a corollary of Theorem 2.5, we have the following bilinear Strichartz estimates by interpolation and summing in dyadic $R$.

Corollary 6.5. Suppose $u_{0}, v_{0}$ are defined as above. Then

- for $\frac{n}{n-1}<q \leq 2$,

$$
\left\|e^{i t \triangle} u_{0} e^{i t \triangle} v_{0}\right\|_{L_{t, x}^{q}\left(\mathbb{R} \times \mathbb{R}^{n-1}\right)} \lesssim M_{1}^{-\frac{1}{2}} M_{2}^{\frac{2 n-1}{2}-\frac{n+1}{q}}\left\|u_{0}\right\|_{L^{2}\left(\mathbb{R}^{n-1}\right)}\left\|v_{0}\right\|_{L^{2}\left(\mathbb{R}^{n-1}\right)} .
$$

- for $2 \leq q \leq \frac{2(2 n-1)}{2 n-3}$,

$$
\left\|e^{i t \triangle} u_{0} e^{i t \triangle} v_{0}\right\|_{L_{t, x}^{q}\left(\mathbb{R} \times \mathbb{R}^{n-1}\right)} \lesssim M_{1}^{-\frac{3}{2 q}+\frac{1}{4}} M_{2}^{\frac{4 n-5}{4}-\frac{2 n-1}{2 q}}\left\|u_{0}\right\|_{L^{2}\left(\mathbb{R}^{n-1}\right)}\left\|v_{0}\right\|_{L^{2}\left(\mathbb{R}^{n-1}\right)} .
$$

- $f$ or $q \geq \frac{2(2 n-1)}{2 n-3}$,

$$
\left\|e^{i t \triangle} u_{0} e^{i t \triangle} v_{0}\right\|_{L_{t, x}^{q}\left(\mathbb{R} \times \mathbb{R}^{n-1}\right)} \lesssim M_{1}^{\frac{n-1}{2}-\frac{n+1}{q}} M_{2}^{\frac{n-1}{2}}\left\|u_{0}\right\|_{L^{2}\left(\mathbb{R}^{n-1}\right)}\left\|v_{0}\right\|_{L^{2}\left(\mathbb{R}^{n-1}\right)}
$$


Remark 6.6. It is clear that, $\forall q>\frac{n}{n-1}, n \geq 3$ and $M_{1}, M_{2} \sim 1$,

$$
\left\|e^{i t \triangle} u_{0} e^{i t \triangle} v_{0}\right\|_{L_{t, x}^{q}\left(\mathbb{R} \times \mathbb{R}^{n-1}\right)} \lesssim\left\|u_{0}\right\|_{L^{2}\left(\mathbb{R}^{n-1}\right)}\left\|v_{0}\right\|_{L^{2}\left(\mathbb{R}^{n-1}\right)},
$$

which improves $q>\frac{n+2}{n}$.

Remark 6.7. When $q=2$ and $n \geq 3$, we have the following sharp estimates for $u_{0}, v_{0}$ defined as above,

$$
\left\|e^{i t \triangle} u_{0} e^{i t \triangle} v_{0}\right\|_{L_{t, x}^{2}\left(\mathbb{R} \times \mathbb{R}^{n-1}\right)} \lesssim M_{1}^{-\frac{1}{2}} M_{2}^{\frac{n-2}{2}}\left\|u_{0}\right\|_{L^{2}\left(\mathbb{R}^{n-1}\right)}\left\|v_{0}\right\|_{L^{2}\left(\mathbb{R}^{n-1}\right)},
$$

which generalizes Bourgain's following estimates to all dimensions

$$
\begin{aligned}
&\left\|e^{i t \triangle} u_{0} e^{i t \triangle} v_{0}\right\|_{L_{t, x}^{2}\left(\mathbb{R} \times \mathbb{R}^{2}\right)} \lesssim M_{1}^{-\frac{1}{2}} M_{2}^{\frac{1}{2}}\left\|u_{0}\right\|_{L^{2}\left(\mathbb{R}^{2}\right)}\left\|v_{0}\right\|_{L^{2}\left(\mathbb{R}^{2}\right)}, \\
&\left\|e^{i t \triangle} u_{0} e^{i t \triangle} v_{0}\right\|_{L_{t, x}^{2}\left(\mathbb{R} \times \mathbb{R}^{3}\right)} \lesssim M_{1}^{-\frac{1}{2}} M_{2}\left\|u_{0}\right\|_{L^{2}\left(\mathbb{R}^{3}\right)}\left\|v_{0}\right\|_{L^{2}\left(\mathbb{R}^{3}\right)}
\end{aligned}
$$

But we remark that Bourgain's estimates are for general $u_{0}$ and $v_{0}$ without the radial assumption, see [2], [4].

\section{References}

[1] Bourgain, J.: Besicovitch type maximal operators and applications to Fourier analysis. Geom. Funct. Anal. 1 (1991), no. 2, 147-187.

[2] Bourgain, J.: Fourier transform restriction phenomena for certain lattice subsets and applications to nonlinear evolution equations. I. Schrödinger equations. Geom. Funct. Anal. 3 (1993), no. 2, 107-156.

[3] Bourgain, J.: Fourier transform restriction phenomena for certain lattice subsets and applications to nonlinear evolution equations. II. The KdVequation. Geom. Funct. Anal. 3 (1993), no. 3, 209-262.

[4] Bourgain, J.: Global solutions of nonlinear Schrödinger equations. American Mathematical Society Colloquium Publications 46. American Mathematical Society, Providence, RI, 1999.

[5] Carleson, L. ANd SJölin, P.: Oscillatory integrals and a multiplier problem for the disc. Studia Math. 44 (1972), 287-299. (errata insert).

[6] Cordoba, A.: The Kakeya maximal function and the spherical summation multipliers. Amer. J. Math. 99 (1977), no. 1, 1-22.

[7] Fefferman, C. And Stein, E. M.: Some maximal inequalities. Amer. J. Math. 93 (1971), 107-115.

[8] Keel, M. and Tao, T.: Endpoint Strichartz estimates. Amer. J. Math. 120 (1998), no. 5, 955-980.

[9] Klainerman, S. and Machedon, M.: Space-time estimates for null forms and the local existence theorem. Comm. Pure Appl. Math. 46 (1993), no. $9,1221-1268$. 
[10] Lee, S. And Vargas, A.: Sharp null forms estimates for the wave equation. Amer. J. Math. 130 (2008), no. 5, 1279-1326.

[11] Moyua, A., Vargas, A. And Vega, L.: Schrödinger maximal function and restriction properties of the Fourier transform. Internat. Math. Res. Notices (1996), no. 16, 793-815.

[12] Moyua, A., Vargas, A. And Vega, L.: Restriction theorems and maximal operators related to oscillatory integrals in $\mathbb{R}^{3}$. Duke Math. J. 96 (1999), no. 3, 547-574.

[13] Stein, E. M.: Some problems in harmonic analysis. In Harmonic analysis in Euclidean spaces (Proc. Sympos. Pure Math., Williams Coll., Williamstown, Mass., 1978), Part 1, 3-20. Proc. Sympos. Pure Math. XXXV. Amer. Math. Soc., Providence, RI, 1979.

[14] Stein, E. M.: Harmonic analysis: real-variable methods, orthogonality, and oscillatory integrals. Princeton Mathematical Series 43. Princeton University Press, Princeton, NJ, 1993.

[15] Stein, E. M. And Weiss, G.: Introduction to Fourier analysis on Euclidean spaces. Princeton Mathematical Series 32. Princeton University Press, Princeton, NJ, 1971.

[16] Strichartz, R. S.: Restrictions of Fourier transforms to quadratic surfaces and decay of solutions of wave equations. Duke Math. J. 44 (1977), no. $3,705-714$.

[17] TAO, T.: Recent progress on the restriction conjecture. In Fourier analysis and convexity, 217-243. Appl. Numer. Harmon. Anal. Birkhäuser Boston, Boston, MA, 2004.

[18] TAO, T.: The Bochner-Riesz conjecture implies the restriction conjecture. Duke Math. J. 96 (1999), no. 2, 363-375.

[19] TAO, T.: Endpoint bilinear restriction theorems for the cone, and some sharp null form estimates. Math. Z. 238 (2001), no. 2, 215-268.

[20] TAO, T.: A sharp bilinear restrictions estimate for paraboloids. Geom. Funct. Anal. 13 (2003), no. 6, 1359-1384.

[21] TAO, T.: A counterexample to an endpoint bilinear Strichartz inequality. Electron. J. Differential Equations (2006), no. 151, 6 pp. (electronic)

[22] TaO, T. And Vargas, A.: A bilinear approach to cone multipliers. II. Applications. Geom. Funct. Anal. 10 (2000), no. 1, 216-258.

[23] TaO, T., Vargas, A. And Vega, L.: A bilinear approach to the restriction and Kakeya conjectures. J. Amer. Math. Soc. 11 (1998), no. 4, 967-1000.

[24] TaO, T., Visan, M. and Zhang, X.: Global well-posedness and scattering for the defocusing mass-critical nonlinear Schrödinger equation for radial data in high dimensions. Duke Math. J. 140 (2007), no. 1, 165-202.

[25] Tomas, P. A.: A restriction theorem for the Fourier transform. Bull. Amer. Math. Soc. 81 (1975), 477-478.

[26] Vilela, M. C.: Regularity of solutions to the free Schrödinger equation with radial initial data. Illinois J. Math. 45 (2001), no. 2, 361-370. 
[27] Wolff, T.: An improved bound for Kakeya type maximal functions. Rev. Mat. Iberoamericana 11 (1995), no. 3, 651-674.

[28] Wolff, T.: A sharp bilinear cone restriction estimate. Ann. of Math. (2) 153 (2001), no. 3, 661-698.

[29] Zygmund, A.: On Fourier coefficients and transforms of functions of two variables. Studia Math. 50 (1974), 189-201.

Recibido: 15 de julio de 2007

Revisado: 23 de abril de 2008

Shuanglin Shao

Department of Mathematics

UCLA

Los Angeles CA 90095-1555, USA

slshao@math.ucla.edu

and

School of Mathematics

Institute for Advanced Study

Princeton, NJ 08540, USA

slshao@math.ias.edu 